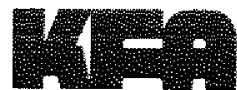

\section{KERNFORSCHUNGSANLAGE JÜLICH GmbH}

Institut für Plasmaphysik

Association EURATOM-KFA

Drift Wave Transport and Origin of the Disruption Phenomenon -

A theoretical Model

by

G. Hasselberg and A. Rogister

Jïl -1830

Februar 1983

ISSN $0366-0885$ 


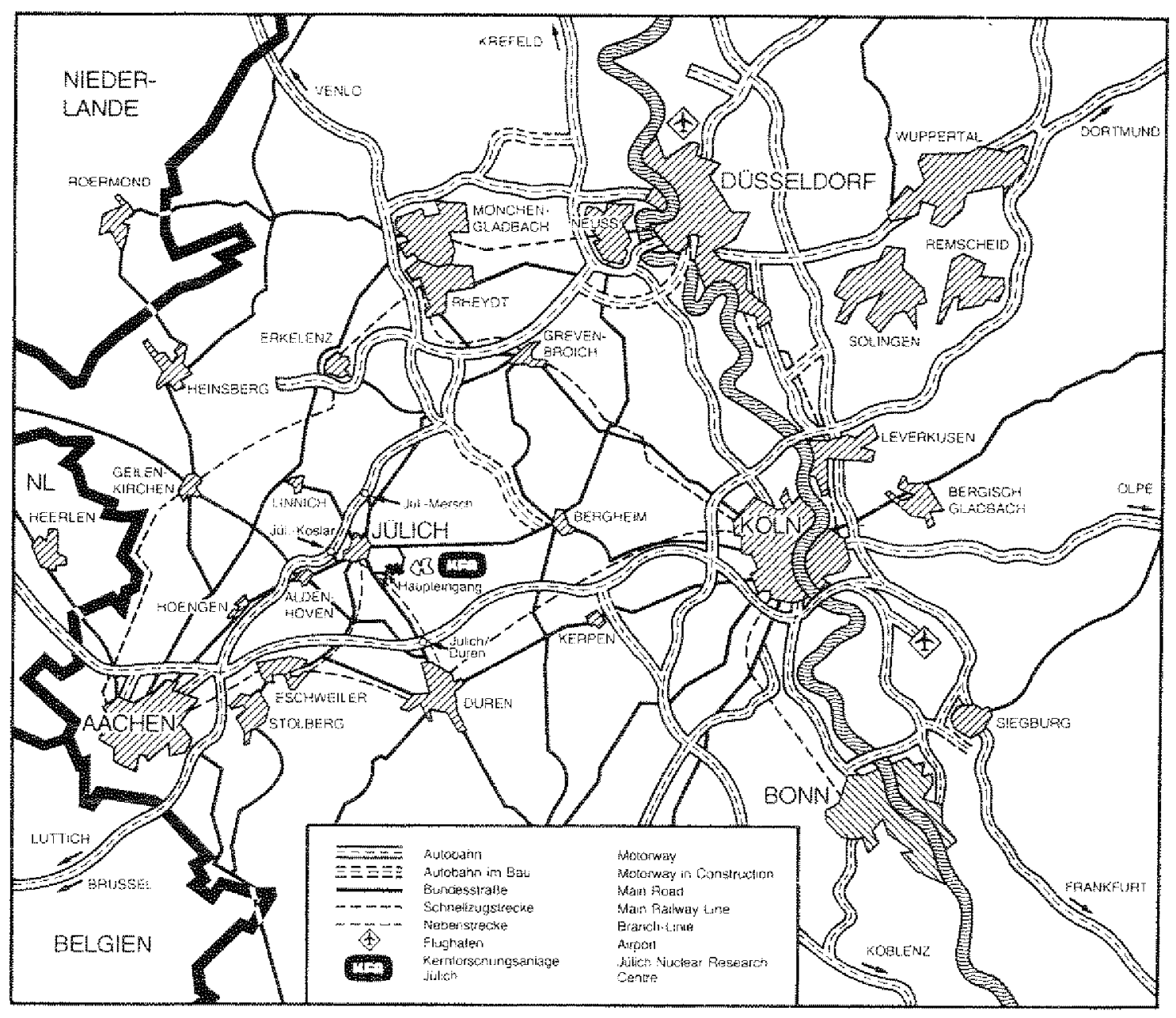

Als Manuskript gedruckt

Berichte der Kernforschungsanlage Jülich - Nr. 1830

Institut für Plasmaphysik Association EURATOM-KFA Jül - 1830

Zu beziehen durch: ZENTRALBIBLIOTHEK der Kernforschungsaniage Jülich GmbH Postfach 1913 - D-5170 Julich (Bundesrepublk Deutschland)

Telefon: $02461 / 610$. Telex: $833556 \mathrm{kfa} \mathrm{d}$ 


\title{
Drift Wave Transport and Origin of the Disruption Phenomenon - \\ A theoretical Model
}

\author{
by \\ G. Hasselberg and A. Rogister
}



DRIFT WAVE TRANSPORT AND ORIGTN OF THE DTSRUPTTON PHENOMENON

- A THEORETICAL MODEL

Günter Hasselberg and André Rogister

Institut fur Plasmaphysik der Kernforschungsanlage Jüich GmbH, Association EURATOM - KFA

5170 Julich, Federal Republic of Germany

Nonlinear ion Landau damping of drift waves yields a splitting of the spectrum into a long and a short wavelength branch. The latter contributes most $(>90 \%$ of the transport and permits to explain the observed relaxation of Tokamak plasma profiles to a weakly unstable state, in the occurence with regard to the dissipative trapped electron mode. The fluxes indeed increase much more rapidly than the 1 inear growth rates. This result and surprising coincidences between the inear theory and empirical laws concerning the high density limit lead us to propose that the slow rise of the sawtooth pulsations of the core occurs whilst the transport in the surrounding layer is insufficient to evacuate the power deposited. The sudden relaxation takes place once the released heat pulses are capable w much as in collisionless shock waves - of exciting the trapped electron mode to a sufficient level to ensure adequate transport. The model explains many experimental features associated with these sawteeth as well as with the related plasma disruptions: contraction of the current channel, high density limit (both the scaling and the order of magnitude are predicted), etc.... 


\section{INTRODUCT TON}

Anomalous plasma transport in magnetic confinement devices is one of the most challenging issues of the controlled thermonuclear fusion programme. The comprehension of the phenomena which are thought to be linked to the nonlinear development of instabilities (1) passes through the steps:

(1) Identification of the linear instability mechanism:

(2) Identification of the nonlinear stabilization mechanism:

(3) Estimation of anomalous transport.

Much of the complexity lies in that several candidates appear under (I) and (2); in addition the theory of the linear eigenmode branches out when the toroidal curvature of the magnetic field is important (2-6). To develop a unified theory taking all of the linear and nonlinear effects rigorously into account would require a tantalizing effort. One is thus bound to consider specific processes and to confront the corresponding theoretical prediction with existing experimental information. The latter concern the turbulence spectra, the heat transport, the confinement of non-recyclable impurities, the existence of an upper density limit, etc. ...

We have show in previous papers (7-9) that nonlinear scattering by ions (nonlinear ion Landau "damping") is the dominant stabilization mechanism of drift instabilities (1) whenever they have a radiative structure à la Pearlstein and Berk (10). The theory indeed then yields turbulence levels which are a factor $L_{s} / L_{n}$ smaller than those obtained wh the ther ion saturation processes (11) and with nonlinear electron processes (12), whereas if the modes have an evanescent structure, owing to toroidal magnetic curvature, nonlinear scattering of ions and of electrons may be competitive mechanisms. $\left(\mathrm{L}_{\mathrm{s}}\right.$ and $\mathrm{L}_{\mathrm{n}}$ are respectively the shear length and the density length scale.) The calculation of Refs. (7-9) introduces sore simplifications: 
i) linear growth and nonlinear damping are treated by perturbation methods and, in the evaluation of the nonlinear terms, the radial eigenfunctions are taken over from linear theory; these assumptions would certainly fail for non-radiative structures.

ii) the wave kinetic equation is obtained in the limit $\mathrm{T}_{i} / \mathrm{T}_{\mathrm{e}} \ll 1$. The latter approximation allows to develop the Bessel function $J_{0}\left(k_{1} a_{i}\right)$ in a Taylor series which introduces considerable algebraic sinplifications. The former is strictly in the line of weak turbulence theory but can in principle be removed since the proper nonlinear radial eigenmode equation has been obtained in Rer. (9). Similarly the wave kinetic equation for the poloidal spectrum, which obtains by imposing certain constraints on the eigenvalue of the radial equation, could be renomalized. Before undertaking these trying improvements of the theory of nonlinear landau damping, we feel it timely, on the one hand, to verify that the crucial hypothesis of a radiative radial structure is consistent with experimental density and temperature profiles and, on the other hand, to confront the predictions of the simplified theory with laboratory results on heat and impurity transport and - rather unexpectedly - on the high density limit.

Ideally, the plasma core where fusion reactions must take place should be screened from the near wall domains, and wall contamination, by an intermediate layer where density and temperature gradients are steep. It is mostly this part of the profile which concerns us here; indeed, closer to the magnetic axis, inside the magnetic surface where the safety factor $q=1$, the anomalous electron heat transport can be semi-quantitatively explained by the sawtooth relaxations of the electron pressure $(13,14)$, whereas atomic processes become dominant closer to the wal1s. Analysis of discharges from TFR (Tokamak Fontenay-aux-Roses) (15) and FT (Frascati Tokamak) (16) have confirmed that the magnetic field curvature is too weak to trap the wave energy. They have also revealed that the radiation losses and the energy 
fed into the modes by the trapped electrons are quite comparable (17). This inmediately suggests that the dissipative trapped electron instability is an essentiai ingredient of plasma transport in Tokanaks, and is indeed so efficient as to prevent strongly unstable profiles to develop. This conclusion is, however, at variance with another inference from experiment, viz. the heat transport calculated on the basis of the neasured turbulence spectra is Iargely insufficient to balance the energy inventory. (The factor 3 reported in (15) is an underestimate since the radial decoupling of the trapped electrons with the modes was not taken into consideration.)

The nonlinear theory that we put forward permits to resolve the paradox which arises here. It predicts on the one hand that one of the two channels for nonlinear ion Landau damping allowed by the dispersion relation, viz. the coupling of two modes with about equal frequencies but different wave numbers, leads to an unexpectedly large energy transfer from long to short wavelengths; the latter are both out of reach of the detection systems used and responsible for most of the anomalous transport. It shows on the other hand that the increase of anomalous transport with linear growth rate is very rapid; hence the apparently universal. character of the relaxation (18). The foregoing implies that drift wave transport cannot be described by scaling laws, and indeed any attempts to do so in the past (11, 19) has led to apparently unsatisfactory results.

The marginal stability criterion of the trapped electron mode is interestingly reminescent of the empirical law for the high density limit $(20,21)$. This has prompted us to propose that the major plasma disruptions and, by extension, the sawtooth relaxations are the consequence of instability quenching and anomalous heat flux clamping at high densities. (The increased collisionality stabilizes the trapped electron mode.) The core then overheats whereas the periphery overcools eventually leading to the excitation of macroscopic instabilities. Release of the excess energy stored in the core (relaxations) will occur when the outgoing heat front is able to lower 
sufficiently the collisionality along its path, increase the growth rate and enhance the transport. This scenario is made plausible by the rapid increase of the anomalous conduction with the growth rate and explains why the observed amplitude and period of the sawteeth increase with density. The gross plasma disruption occurs either as a limiting relaxation of the core whereby the energy released drives the plasma out of equilibrium or as a limiting constriction of the current chamel whereby magnetic island overlap occurs. The high density limit derived in this way is in remarkable agreement with experiments. In a companion paper devoted to the related problem of impurity transport, it will be shown that the theory also brings a plausible explanation to the cleaning action of the sawteeth.

The present research is organized as follows. We recall in Section II the Iinear theory of the dissipative trapped electron mode, the nonlinear theory of the saturation process, and the theory of the related transport. We discuss the experimental profiles in section III and present the argunents which favour the hypothesis, on which the theory relies, of a wave radiating structure. We compare in Section IV the experimental and theoretical electron heat transport; we also justify theoretically the observed near-marginal stability of the experimental profiles. We discuss in Section $V$ the origin of the sawtooth relaxations and of the gross plasma disruptions; we compare the predictions of our model with experimental information. We briefly summarize our results in section VI.

\section{THEORETICAL PRELIMINARTES}

A. Review of Linear Theory

The "non-adiabatic" (n-a) component of the density fluctuation releases the energy available to drive the instability. (The consecrated but illchosen wording "non-adiabatic" designates the component out of phase with the potential $\phi$.$) Retaining only the contribution from the trapped electrons,$ we have: 


$$
\left[\tilde{n}_{\ell, m}(x)\right]_{e}^{n-a}=i(2 / \pi)^{1 / 2} \varepsilon_{0}^{3 / 2} \frac{e^{-w} e, e^{\left(1+3 n_{e} / 2\right)}}{v_{e i}\left(1+z^{-1}\right)} \sum_{p} \phi_{l, m+p}(x) s_{\ell}(p, x)
$$

$x=r-r_{l, m}$ is the distance from the mode rational surface $r_{\ell, m}$ which is defined by the relation $\mathrm{lq}\left(\mathrm{r}_{\ell, \mathrm{m}}\right)+\mathrm{m}=0\left(\mathrm{~m}=\mathrm{rk}_{\theta}\right.$ : poloidal mode number; $\ell:$ toroidal mode number). $\phi_{\ell, m^{*} \mathrm{p}}$ is the coefficient function of the $\mathrm{p}-\mathrm{th}$ bounce frequency harmonic of the normalized potential $e \Phi / T$. Transtation invariance requests that $\phi_{\ell, m}(x)=\exp (-i p \pi) \phi_{l, m}\left(x+p \Delta_{\ell}\right)$ where $\Delta_{\ell}=r_{\ell, m+1}-r_{\ell, m}=\left(k_{\theta} \sigma\right)^{-1}$ is the distance between neighbouring rational surfaces and $\hat{\sigma}=(\mathrm{rdenq} / \mathrm{dr})_{r_{\ell, \mathrm{m}}}$ is the local shear parameter. The phase factor exp(-ipt) is chosen to give maximum interference of the hamonics $\exp [i(m+p) \theta] \phi_{,} m+p(x)$ at the angular location $\theta=\pi$ where the population of trapped electrons is largest $(\theta=\pi$ corresponds to the outer boundary of the equatorial cross section of the torus). The function $S_{2}(p, x)$ accounts for the radial decoupling of the trapped electrons wh the modes; in the approximations of a pitch angle collision operator and of a parabolic tagnetic we1 (22).

$$
S_{\ell}(p, x)=\langle\alpha\rangle_{\theta} \int_{0}^{\pi} d z z \frac{J_{1}\left[\left(p+x / \Delta_{\ell}\right) z\right]}{p+x / \Delta_{\ell}} \frac{J_{1}\left[\left(x / \Delta_{\ell}\right) z\right]}{x / \Delta_{\ell}}
$$

where $J_{1}$ is the Bessel function of the first kind. The model, however, systematicaliy overestimates the response of the electrons and actually yields the perturbed density for $\theta=\pi$. Noting that the maximum velocity of trapped particles along the field lines is proportional to $(1-\cos \theta)^{1 / 2}$ (at a given energy) and that their detrapping collison frequency vary as $(1-\cos \theta)^{-1}$, we have introduced a correction factor $\alpha(\theta)=(1-\cos \theta) / 2)^{3 / 2}$, or rather

$$
\left.\cos \int_{\theta}^{0}(\mathrm{~d} \theta / \pi)(1-\cos \theta) / 2\right]^{3 / 2}=4 / 3 \pi
$$

since the poloidal structure is not taken into account in the theory. It 


\section{2}

can be shown that Eq. (26) now agrees (within $10 \%)$ with the corresponding equation (26) of Reference (23) for $x=0$ in the 1 imit of isolated rational surfaces. The remaining notations introduced in Eq. (2a) are $w_{e, \ell}^{*}=\ell c T_{e} / e_{i} L_{N} R B_{\theta}=-k_{\theta} a_{s} c_{S} / L_{N}$ for the electron diamagnetic frequency, $\varepsilon_{0}=r / R$ for the inverse aspect ratio, and $\eta_{e}=d \operatorname{lnT} \mathrm{e}_{\mathrm{d}} / \mathrm{hnN}$; the collision frequency is defined as $\nu_{e i}=3 \sqrt{\pi} / 4 \tau_{e}$ where $\tau_{e}$ is the standard collision time of Bragisnkii (24); $\mathrm{Z}$ is the effective ion charge, and $z^{-1}$ ei a good approximation of the electron contribution to the scattering; the sound velocity $c_{S}=\left(T_{e} / m_{i}\right)^{I / 2}$ and $a_{s}=c\left(T_{e} m_{i}\right)^{1 / 2} / e_{i} B ; L_{N}^{-1}=d \operatorname{lnN} / d r$. The result $(2 \pi)$ obtains if the inequalities

$$
\omega-\bar{\omega}_{d, e}<\left(1+z^{-1}\right) v_{e i} / \varepsilon_{0}<\bar{w}_{b, e}
$$

are satisfied where $\omega_{e, \ell}^{-d}=-\ell c T_{e} / e_{i} R^{2} B_{\theta}$ is the time-average magnetic drift frequency and $\omega_{e}^{-b}=\sqrt{\varepsilon_{0}} c_{e} / q R$ the electron bounce frequency. The left hand-side inequality is well verified for the profiles we consider whereas generalization to finite values of the collisionality parameter $v^{*}=\left(1+2^{-1}\right)$ $v_{e} q^{R / \sqrt{2} \varepsilon_{o}^{3 / 2}} c_{e}$ is obtained by replacing

$$
\omega-\omega_{e}^{*}\left(1+3 n_{e} / 2\right) \rightarrow\left(\omega-\omega_{e}^{*}\right) a_{n}\left(v^{* 1 / 2}\right)-(3 / 2) \eta_{e} e_{e}^{*} b_{n}\left(v^{* 1 / 2}\right)
$$

The factors $a_{n}(t)=e^{-t}\left(1+t+t^{2}\right)$ and $b_{n}(t)=e^{-t}\left(1+t+t^{2} / 2+t^{3} / 3\right)$ account for the fact that low energy electrons, not being able to describe a full orbit between the mirroring points because of enhanced scattering, must be substracted from the non-adiabatic response though they are temporarily on a trapped trajectory.

The evolution of the W.K.B. wave

$$
\Phi_{\ell, m}(x, t)=\bar{\Phi}_{\ell, m}(x, t) \exp \left[i \int_{0}^{x} \bar{k}_{r}\left(x^{i}\right) d x^{\ell}-i \omega_{\ell} t\right]
$$


where the bars merely indicate when the variations in space or time are "slow" (in the sense of Krylow and Bogoliubov (25)) is defined through the equation (9)

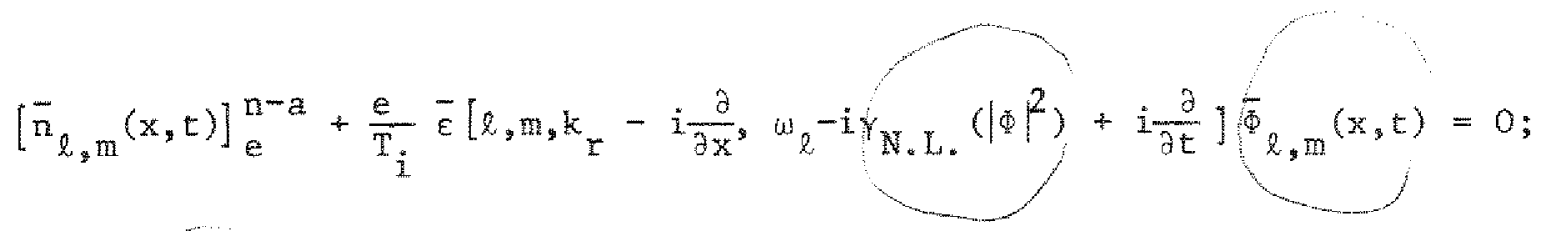

the operator Y.L. of course describes the nonlinear coupling phenomena and $\bar{E}$ is the dispersion functional. Within the framework of the approximation introduced previously, the form

$$
\bar{\varepsilon}\left(l, m, k_{r}, \omega_{l}\right)=\frac{T_{i}}{T_{e}}\left[1-\frac{\omega_{e}^{*} l}{\omega_{l}}+\frac{\left(k_{r}^{2}+k_{\theta}^{2}\right) c_{s}^{2}}{\Omega_{i}^{2}}-\frac{\bar{k}^{2} c^{2} s}{\omega^{2}}\right]=0
$$

is suitable. The frequency and radial node number of the zero-damped mode (26) are consequently $\omega_{\ell}=\omega_{e, \ell}^{*} /\left(1+k_{0}^{2} a_{s}^{2}\right)$ and $k_{r}=-\left(\Omega_{i} / \omega_{\ell}\right)\left|k^{*}\right| x$ respectively; the choice of sign in the latter expression is imposed by causality and corresponds to the radiating solution: $\left(v_{g}\right)_{r} \equiv-(\partial E / \partial K) /(\partial E / \partial \omega)>0$; the parallel mode number is $k_{n}=\vec{k} \cdot \vec{B} / B=\left(\ell B_{\phi} / R B\right)$ (dlnq $\left./ d r\right) \times$.

The eigenvalue equation corresponding to the most unstable wave function

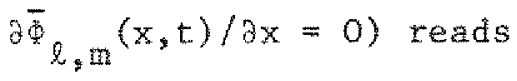

$$
\gamma \equiv \frac{\partial}{\partial t} \ln \bar{\Phi}_{l, \mathrm{~m}}(t)=i \frac{\mathrm{T}_{i}}{e}\left(\frac{\partial \varepsilon_{\mathrm{g}}}{\partial \omega}\right)^{-1} \frac{\left\langle\Phi \mid \mathrm{n}_{\mathrm{e}}^{\mathrm{n}-\mathrm{a}}\right\rangle}{\langle\Phi \mid \Phi\rangle}-\frac{1}{2} \frac{\partial}{\partial \mathrm{x}}\left(v_{\mathrm{g}}\right)_{\mathrm{r}}+\gamma_{\mathrm{NL}}\left(|\Phi|^{2}\right)
$$

with the notation $\langle\Phi \mid \psi\rangle \equiv \int_{l x}^{\infty} d x \Phi_{l, m} \psi, m$. Note that the radiating solution is spatially bounded as linear ion absorption takes place far away from the rational surface; ion absorption gives also rise to the shear damping term $-(1 / 2) \partial\left(v_{g}\right) \mathrm{r} / \partial \mathrm{x}=-\left|\mathrm{k}_{i}\right| c_{\mathrm{s}}^{2} \omega / \Omega_{i} \omega_{\mathrm{e}}^{*}$ in $\mathrm{Eq}$. (5b). For the destabilizing contribution $i<\Phi \mid n_{e}^{n-a}>/\langle\phi|$ we shall use an interpolation formula joining the asymptotic forms which obtain when $\left(\mathrm{x}_{t} / \Delta\right)_{Q}>>$ and $\left(\mathrm{x}_{t} / \Delta\right)_{\ell}</\left|\mathrm{x}_{t}=\right| \partial \mathrm{k}_{\mathrm{r}} /\left.\partial \mathrm{x}\right|^{-1 / 2}$ is the 
absolute value of the point of stationary phase of the W.K.B. potential

(Eq. (4). In the first case, $\left\langle\phi_{\ell, m} \mid \sum_{p} \phi_{l, m+p} S_{p}\right\rangle=\langle\alpha\rangle_{\theta} 2 \pi^{2} \Delta_{l} \phi_{l, m}(0)^{2}$ (see Reference $(22)$, whereas in the second $\left\langle\phi_{\ell, \mathrm{m}} \mid \sum_{\mathrm{p}} \phi_{\ell, \mathrm{m}+\mathrm{p}} \mathrm{s}_{\mathrm{p}}\right\rangle=\langle\alpha\rangle_{\theta}$ $\left(\pi^{4} / 16\right)<\phi_{\ell, m} \mid \phi_{\ell, m}>$. Hence the interpolation formula for the normalized linear growth rate:

$$
\tilde{\gamma}_{L}=\mu c\left[1-\exp \left(-\lambda \Delta / x_{t}\right)_{l}\right]\left(1+d_{D} \frac{y}{1+y}\right) \frac{\sqrt{y}}{1+y}-1
$$

where

$$
c=\langle\alpha\rangle_{\theta}\left(\frac{\pi}{2}\right)^{7 / 2}\left(\frac{m_{e}}{2 m_{i}}\right)^{1 / 2} \frac{L_{s}^{2}}{L_{n}^{2}} \frac{3}{2} \eta_{e} \sigma v^{*-1} b_{n}\left(v^{* 1 / 2}\right)
$$

and $d_{n}=2 a_{n} / 3 n_{e} b_{n}$. With the present model of a pitch angle colisision operator, we obtain $\lambda=2 \pi^{2} \phi^{2}(0) /\left(\pi^{4}\langle\phi \mid \phi\rangle / 16 x_{t}\right)=2.59$ whereas a scalar model would yield $\lambda=\pi / 2$ (27). The factor 4 has been introduced in order to check whether the theoretical and experimental results can be fitted given the imprecisions inherent to the analytical development.

B. Review of Nonlinear Theory

The enhancement of drift waves out of thermal noise is possible because the phase velocity along the magnetic field appreciably exceeds the ion thermal velocity: the resonant ion population and the linear absorption of wave energy are then limited. The beat of two fast modes, however, can exchange energy with the bulk of the ion population if the beating frequency is sufficiently small, i.e. $\left|\omega-\omega^{8}\right|<\left.\right|^{\prime}|,| \omega^{\prime} \mid$, that is if the frequencies of the original modes are sufficiently close, $i . e,\left|1-\omega^{8} / \omega\right| k<$. This process, known as nonlinear Landau damping, includes both Compton scattering and diffraction by the shielding cloud (or induced scattering); in contrast 
to single-mode marginal stability theories (28), it is compatible with an evolution of the instability towards a non-singular saturated state. It has first been invoked by Sagdeev and Galeev (29) in relation to drift waves and by the authors (30) in connection with parametric heating of plasmas. However, it was not recognized that the condition $\left|1-w^{\prime} / w\right|<<$ could be exploited to eliminate the integral character of the wave kinetic equation. A detailed description of the procedure is given in (9). This yields for the problem at hands

$$
y \frac{\partial f}{\partial y}+\frac{2-y}{1-y} f+(2-y)^{2} \frac{\partial f^{\dagger}}{\partial y}+\frac{2-y}{1-y} f^{\dagger}=s
$$

if the approximations (i) and (ii) listed in Section I are introduced and $y=k_{\theta}^{2} a_{s}^{2}$. The functions $f \equiv f(y)$ and $f^{\dagger} \equiv f(1 / y)$ are related to the onedimensional poloidal spectrum of the normalized adiabatic density fluctuation

$$
\begin{aligned}
\left\langle\left|\tilde{n}_{\mathrm{m}}\right|^{2}\right\rangle_{r_{0}}=\left\langle\left|\phi_{\mathrm{m}}\right|^{2}\right\rangle_{r_{0}} & =\sum_{\ell}(\delta r)^{-1} \int_{r_{0}-\delta r / 2}^{r_{0}+\delta r / 2} d r\left|\phi_{\ell, \mathrm{m}}\left(r-r_{\ell, m}\right)\right|^{2} \\
& =\left.\Delta_{\mathrm{m}}^{-1} \int_{-\infty}^{\infty} d x\right|_{\mathrm{m}}\left(x,\left.r_{0}\right|^{2}\right.
\end{aligned}
$$

$\left(\Delta_{\mathrm{m}}=q \Delta_{\ell}=q /\left|\mathrm{k}_{\theta}\right| \hat{\sigma}\right)$ by the relation

$$
f(y)=(2 \pi)^{-1}(q / \hat{\sigma})\left\langle k_{\left.r^{3} a^{4}\right\rangle}^{4}\left\langle\left|\tilde{n}_{m}\right|^{2}\right\rangle_{0}\right.
$$

where $\left(\mathrm{k}_{\mathrm{r}}^{4} \mathrm{a}_{\mathrm{s}}^{4}\right)^{1 / 2}$ is a measure of the radial width of the $2-\mathrm{D}$ spectrum. If one assumes that the spatial decay induced by linear ion absorption is of the form $\left|\phi_{m}(x)\right|^{2} v \exp \left(-x^{2} / x_{t}^{2}\right)$, one obtains $\left\langle k_{r}^{4} a_{s}^{4}\right\rangle=3 a_{s}^{4} / 4 \varepsilon^{2} x_{t}^{4}$. The quantity $\varepsilon \ll 1$ will be evaluated in section IIC. 
The source term in Eq. (8) is

$$
s \equiv-\operatorname{sign}(1-y) \frac{\tilde{\gamma}_{L}-\tilde{\gamma}}{\Delta_{0}} \frac{(1-y)^{2}}{(1+y)^{3}} y^{-3 / 2}
$$

where

$$
\Delta_{0}=24 \pi^{2} \tau^{2} \operatorname{Rr}\left|L_{N}\right| / a_{S}^{3}
$$

$\mathrm{R}$ is the major radius of the torus, $\tau=\mathrm{T}_{i} / \mathrm{I}_{e}$, and $\tilde{\gamma}_{L}$ - respectively $\tilde{\gamma}-$ is connected to the linear growth rate - respectively the nonlinear damping rate - via

$$
\frac{\gamma_{L}}{\tilde{\gamma}_{L}}=\frac{Y}{\tilde{\gamma}}=|\omega| \frac{\left|L_{N}\right|}{\left|\tilde{L}_{S}\right|}
$$

$\left(\mathrm{L}_{\mathrm{s}} \equiv \mathrm{qR} / \widehat{O}\right.$ is the shear length). It is trivial to note that a suprathermal spectrum should be marginally stable, i.e. $\tilde{\gamma} \equiv$ o where $f>0$; conversely a spectrum at the thermal level (neglected) must be damped, $i . e . \tilde{\gamma} \leq 0$ where $f=0$. Mathematically, negative spectra f<0 in certain strips can be avoided only by ascribing negative values to those are obtained from the constraint $f \equiv 0$ in the strips.

The first two terms on the left-hand side of Eq. (8) arise from the beating of modes with neighbouring poloidal mode numbers $k_{\theta} \approx k_{\theta}$; through this "close interaction" process energy cascades to ever longer wavelengths if $k_{\theta} a_{s}<1$, and to ever higher mode numbers if $k_{\theta} a_{s}>1$. The change of sign of the group velocity $\partial \omega / \partial \mathrm{k}_{\theta}$ for $\mathrm{k}_{\theta}{ }^{a_{S}}=1$ explains this different behaviour. The last two terms on the left-hand side occur because the same frequency $w$ pertains to the modes with wave numbers $k_{\theta}$ and $k_{\theta}^{+}=1 / k_{\theta} a_{s}^{2}$; energy is transferred from long $\left(k_{\theta} a_{s}<1\right)$ to correspondingly short $\left(k_{\theta}^{+} a_{s}>1\right)$ wavelengths through this "distant interaction" process. It should be noted here that 
the transfer term considered previously in References (29) and (31) was shown in (9) to be negligible in the limit $t \rightarrow 0$.

Equation (8) has been solved analytically for arbitrary linear growth rates, but in the present paper the discussion 11 be centered on the dissipative trapped electron instability. We recall that the solution shows the source term to play always an insignificant role at large $y^{\prime \prime} s^{\prime}$ this is a consequence of the rapid increase of nonlinear ion Landau damping with this parameter; actually the energy input via distant interaction and the outflow via close interaction completely determine the spectrum at sufficiently high poloidal mode numbers. The onset of the transfer occurs for $y \cong y_{1}=0.382$ (and correspondingly $y \cong y_{2}=y_{1}^{-1}=2.62$ ) at the expense of the energy content in the long wavelengths spectrum $(7-9)$. The latter ought then to be maximm for ${ }_{\theta}{ }^{a}{ }_{s}=0.62 ;$ this result agrees with experimental values reported from ATC (Adiabatic Toroidal Compressor (32) TFR (33) and PIT (Princeton Large torus) (34); only from Alcator have lower values been systematically reported (35). (The values $y=y_{1}$ and $y=y_{2}$ correspond to regular singular points of the second order differential equation that one can derive from (8) by elimination e.g. of $\mathrm{f}^{\dagger}$.)

As a consequence of $\mathrm{Eq}$. (8), the spectrum at large node numbers is of the form $\left\langle\left|\tilde{n}_{\mathrm{n}}\right|^{2}\right\rangle_{\mathrm{o}} \mathrm{s} \mathrm{k}_{0}^{-4}(9)$ to be compared with the Micrator observations $k_{0}^{-3.5}$ (36) in low ion temperature plasma, It can be shown (9) that the spectral index $(=4)$ is compatible with the hypothesis of an inertial subramge, similar to Obukhofe's in Navier stokes turbulence $(37)$, in which only energy cascade occurs.

\section{Revies of Transport Theory}

The general expressions for particle and heat transport are given in Fquations (36) and (37) of Reference (22) where the angulat brackets reprem sent, as in $\mathrm{Eq} .(9 \mathrm{a})$, spatial averages over distances 5 which are intermediate between the macroscopic and microscopic length scales of interest:

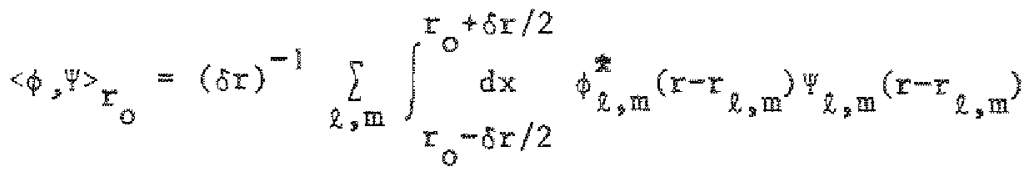

$$
\begin{aligned}
& =\sum_{\mathrm{m}} \Delta_{\mathrm{m}}^{-1} \int_{-\infty}^{\infty} \mathrm{dx} \phi_{\mathrm{Z}}^{\mathrm{x}}\left(\mathrm{x}, \mathrm{r}_{\mathrm{O}}\right) \Psi_{\mathrm{H}}\left(\mathrm{x}, \mathrm{x}_{0}\right)
\end{aligned}
$$


The fluctuations of electron pressure and density having a similar radial dependence, it is sufficient to evaluate the expression

$$
\begin{aligned}
&\left\langle\phi_{\mathrm{m}}^{*}, \sum_{\mathrm{p}} \phi_{\mathrm{m}+\mathrm{p}} \mathrm{S}_{\mathrm{p}}\right\rangle_{\mathrm{r}_{0}}=8\langle\alpha\rangle_{\theta} q^{-1}\left|\phi_{\mathrm{m}}(0)\right|^{2} \int_{0}^{\pi / 2} \mathrm{du} u \int_{-\infty}^{\infty} \mathrm{dt} \sum_{\mathrm{p}} \\
& \exp \left[i p t^{2}\left(\Delta / 2 \mathrm{x}_{\mathrm{t}}\right)_{\ell}^{2}\right] \frac{\mathrm{J}_{1}[(\mathrm{t}+\mathrm{p}) \mathrm{u}] \mathrm{J}_{1}[(\mathrm{t}-\mathrm{p}) \mathrm{u}]}{\mathrm{t}^{2}-\mathrm{p}^{2}}
\end{aligned}
$$

The exponential can be approximated by unity since either $\left(\Delta / x_{t}\right) \ll l$ (the mode overlaps many rational surfaces) or the $p=0$ term $i$ dominant when $\left(\Delta / x_{t}\right)_{\ell}>1$; the second integral can thus be taken from Watson (38) and the sum obtained by the method of residues (Appendix $A$ ). We obtain $\left.{ }_{0} \ldots\right\rangle_{0}$ $=2 \mathrm{~g} \mathrm{r}^{2}\langle\alpha\rangle_{\theta} q^{-1}\left|\phi_{\mathrm{m}}(0)\right|^{2}$ where $4 / 9 \leq \mathrm{g} \leq 1$, the lower bound corresponding to the asymptotic limit $\Delta / x_{t}>>1$ and the upper bound to $\Delta / x_{t}<<$; we shall adopt the interpolated form $g=\left(1+4 \Delta^{2} / 9 x_{t}^{2}\right) /\left(1+\Delta^{2} / x_{t}^{2}\right)$.

In order to relate the squared amplitude $\left|\phi_{\mathrm{m}}(0)\right|^{2}$ and the spectral function $f(y)$, we assume that the spatial decay of the radial eigenmode is properly taken into account by multiplying the W.K.B. phase factor in Eg. (4) by $\left(1+i \varepsilon k_{K} /\left|k_{x}\right|\right), \varepsilon<<1$. Hence from $\mathrm{Eq} .(9 \mathrm{~b})$ :

$$
\left|\phi_{m}(0)\right|^{2}=\left|n_{m}(0)\right|^{2}=\frac{8 \pi^{1 / 2}}{3} \varepsilon^{5 / 2} \frac{x_{t}^{3}}{a_{s}^{3}} y^{-3 / 2} f .
$$

To obtain $\varepsilon$ quantitatively, we request that $e x_{c}^{2} / x_{t}^{2}=1$ where the cut-off lengthscale $x_{c}$ is defined as the distance from the rational surface for which the Landau residue and the parallel pressure contribute equally to the dielectric function in its kinetic form. More precisely, we request $\mid$ Re $Z^{*}\left(\omega / \sqrt{2} k_{1}^{*} x_{c} c_{i}\right)|=| \operatorname{In} Z^{*}\left(\omega / \sqrt{2} k_{p}^{q} x_{c} c_{\dot{i}}\right) \mid$ where $Z$ is the plasma dispersion function (39) and obtain $w / \sqrt{2} k_{i}^{*} c_{i} \cong 1.5 \mathrm{x}_{\mathrm{c}}$. Recaling the definition of $x_{t}^{2}=\left|\partial k_{r} / \partial x\right|^{-1}=\left|\omega / k_{v i}^{*} \Omega_{i}\right|$, there follows that 


$$
\varepsilon=4.5 \tau(1+y)\left|L_{n}\right| /\left|L_{s}\right|
$$

Note that the cut-off mode number is given by $k_{r c}^{2}=1 / \varepsilon x_{t}^{2}=1 / 4.5 a_{i}^{2}$. Applying successively the transformation (11) and (12) to the electron heat transport equation yields

$$
\begin{aligned}
& \frac{3}{2} \frac{\partial \mathrm{P}}{\partial \mathrm{t}}=-\frac{1}{\mathrm{x}} \frac{\partial}{\partial \mathrm{x}} \frac{\mathrm{cT}}{\mathrm{eB}}\left|\frac{\mathrm{dq}}{\mathrm{dr}}\right| \Gamma_{\mathrm{m}} \mathrm{i} \mathrm{m}|\mathrm{m}| \int_{-\infty}^{\infty} \mathrm{dx} \phi_{\mathrm{m}}^{*} \frac{3}{2} \rho_{\mathrm{e}, \mathrm{m}} \\
& -\tau_{e}\left|\frac{d q^{-1}}{d r}\right| \sum_{m} i m|m| \int_{-\infty}^{\infty} d x \phi_{m}^{*} n_{m} \\
& +E J-P_{e i}-P_{\text {rad. }}-\ddot{P}-P_{0} \\
& =\left(\frac{1}{r} \frac{\partial}{\partial r} r-\frac{\nu}{L_{n}}\right) \frac{5 \mu\langle\alpha\rangle}{3} \frac{m_{e}}{m_{i}}-\frac{\tau^{1 / 2}}{k_{1}^{5} a_{i}^{5}} v^{*-1} b_{p}\left(v^{* 1 / 2}\right) G_{1} \frac{a_{s}^{2} c_{s}}{L_{s}} N \frac{\partial T_{e}}{\partial r} \\
& +E J-P_{e i}-P_{\text {rad. }}-\stackrel{M}{P}-P_{0}
\end{aligned}
$$

(To obtain the equation for particle transport, aN/at $=\ldots$ set $v=0$ and replace $\left.b_{p}, 5<\alpha\right\rangle_{\theta} / 3$, and $0.6 d_{p}$ (appearing in Eq. (15a)) by $b_{n},\langle\alpha\rangle_{\theta} / 3$, and $d_{n}$ respectively. E and $J$ are the toroidal electric field and current density whereas $P_{e i}, P_{\text {rad }}, P$, and $P_{o}$ are the power losses through the ion channel, radiation, sawtooth relaxations, and other unidentified processes, e.g. instabilities specific to the otter plasma layers. The term $-v \ldots$ represents an additional heat exchange from the electrons to the ions induced by the turbulence, and the parameter $v=G_{0} b_{n}\left(v^{* 1 / 2}\right) / 5 G_{1} b_{p}\left(v^{* 1 / 2}\right)$ where

$$
\begin{aligned}
& G_{1}=\int_{0}^{\infty} \frac{d y}{y}(1+y)\left(1+0.6 d \frac{y}{1+y}\right) g\left(f \Delta_{0}\right), \\
& G_{0}=\int_{0}^{\infty} \frac{d y}{y}\left(1+d_{n} \frac{y}{1+y}\right) g\left(E_{\Delta_{0}}\right)
\end{aligned}
$$


$b_{p}(t)=a_{p}(t)+t^{4} e^{-t} / 15, a_{p}=2 a_{p} / 3 n_{e} b_{p}$, and $a_{p}(t)=e^{-t} \sum_{0}^{3} t^{n}|n|$

III. REMARKS ON THE EXPERIMENTAL RROFTLES.

In this Section, we verify that the experimental profiles of density and temperature (Fig. 1) rule out the hypothesis of wave energy trapping by the field curvature. This result is important since it enables to restrict considerabiy the range of conjectures in regards to the possible mechanisms of excitation;

it justifies also the development of the nonlinear theory on the basis of radiative structures. We show further that the influence of electron inertia and curvature drifts on the stability are negligible.

The radial eigenfunctions will be localized, and shear damping impeded, if the neighbouring "quasi-modes" $(\ell, m),(\ell, m+1), \ldots$ are strongly coupled through toroidal effects, especially ion curvature drifts. Theoretical considerations (3-5) show that an approximate localization criterium is

$$
\frac{(1+y)^{2}}{y^{2}} \ll \frac{q^{2} \hat{\sigma}^{2}}{\varepsilon_{N}^{2}}\left(\frac{\left|\varepsilon_{N}\right||1-2 \hat{\sigma}|}{\sigma^{2}} \frac{1+y}{y}-1\right)
$$

where $\varepsilon_{\mathbb{N}} \equiv \mathrm{L}_{\mathrm{N}} / \mathrm{R}$. We add that in this version of the theory, positive interference of the components $\exp [i(m+p) \theta] \phi_{h} m+p(x), p=0, \pm 1, \ldots$ - i.e. "ballooning" - occurs on the inside of the torus if $\hat{\sigma}>1 / 2$, and on the outside if $\hat{0}<1 / 2$, provided that $\varepsilon_{\mathbb{N}}<0$ which corresponds to nomal density profiles. From the inequation (16a) one can obtain the curves (Fig. 2) limiting, as a function of radius, the range of poloidal mode numbers for which shear damping partially or fully cancels. Letting $\mathrm{t} \equiv\left|\epsilon_{\mathrm{N}}\right||1-2 \hat{\theta}| / \vec{\sigma}^{2}$ and $s \equiv \varepsilon_{\mathbb{N}}^{2} / q^{2} \sigma^{2}$, they are defined by

$$
\frac{y}{1+y}=\frac{t}{2} \pm \sqrt{\left(\frac{t}{2}\right)^{2}-s} .
$$


If $t^{2} \leq 48$, $\dot{1} . e$. if $|1-2 \hat{\sigma}| \leq 2 \hat{\sigma} / q$, the radiative structure prevails for all mode numbers; this is always the case at intermediate radii where $\hat{\sigma} \simeq 1 / 2$. In the vicinity of the magnetic axis, where $\hat{\sigma} \rightarrow 0$ and the distance between rational surfaces increases without limit, the right hand-side of Eq. (16b) is larger than unity and again the radiative structure prevails. In the outer regions of the plasma there is a small range of poloidal mode numbers for which wave trapping may occur. Ballooning however then takes place on the high field side of the torus where the population of trapped particles is smaller than average whilst their scattering frequency is larger. Since moreover the corresponding wavelengths are very large (see Fig. 2) we conclude that the non-adiabatic density fluctuation of trapped electrons is here negligible.

With the reservation that the near-axis region may deserve refined theoretical developments (given the small gradients) the latest theories then predict both the collisionless (40) and the collisional (41) drift waves to be absolutely stable in the dischages considered except perhaps in the case of the Frascati Tokanak where there is, at small radii $(0.175<p<0.375)$, a window of mode numbers in the

range $k_{\theta} a_{s}=1$ for which shear damping is ineffective. We recall that collisional drift modes would especially be appropriate to the periphery where the plasma is in the Pfirsch-Schluter regime $\left(\nu^{*}>e^{-3 / 2}\right)$; the collisionless instability would be appropriate to the core where the plasma is in the plateau regime $\left(e^{-3 / 2}>v^{ \pm}>1\right)$. Positive values of $n_{e}=d \operatorname{lnT} e^{/ d \ell n N}$ have for these modes - and in contrast to the trapped electron mode - a stabilizing influence on the non-adiabatic electron response.

It has been suggested that the trapped electron mode will stabilize when the effective collision frequency becones smaller than the wave frequency (42) except for a narrow range of wave numbers for which electron curvature drift resonance takes place (43). Fig. 3a shows that the ratio $(e w / v e i) y=1$ hardly reaches $2 \cdot 10^{-1}$ across the profiles that we have analysed. 
This precludes both of the collisonless processes just described. The curve (Fig. 3b) of $\left(\varepsilon \bar{w}_{d} / v_{e i}\right)_{y=1}$ versus the nomalized radius $p=r / a$ (a is the limiter radius) is also given in order to justify the neglect of the time average magnetic drift frequency in the calculation of the trapped electron response (Section IIB).

Sumarizing, the dissipative trapped electron mode appears to be the only possible cause of drift turbulence. It is well described by Eqs. (1) and (7) for the profiles we consider.

Fig. 4 gives the geometrical Iimits $\rho_{1}$ and $\rho_{2}$ of the layer where this mode is excited as functions of the parameters $\lambda$ and $\psi$ appearing in Eq. (7a). $\left(\rho_{1,2}\right.$ of course obtain from the equations $\gamma(\rho, \lambda, \mu, y)=0$ and $\partial y(\rho, \lambda, \mu, y) /$ $\partial y=0$ ) and Fig. 5 shows the maximum growth rate labelled by the corresponding mode numbers for a selected value of $\mu$ and $\lambda=2$. From Fig. 4 one observes that the existence of a turbulent zone requires ascending values of these parameters, in the order, for the TFR moderate and high density discharges and for the $\mathrm{FT}$ discharge. This sequence corresponds to increasing densities

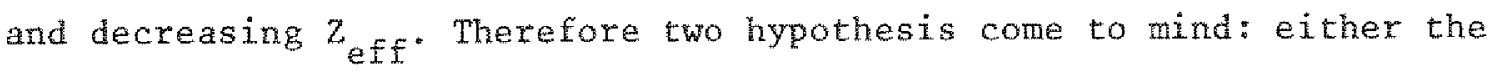
impurities are not uniformly distributed as assumed but their concentration goes up with radius, or the power released intermittently by the sawteeth is sufficient to lower the collisionality and hence increase the growth rate and the transport by micxoturbulence for a short time. In the first altemative the effective charge in the transition layer would increase proportionally to the average degree of contamination and readjust accordingly the growth rates of the three discharges. The second alternative is theoretically plausible in view of the calculated rapid increase of the turbulent transport with the growth rate (Sec. IV). It is experimentally consistent with the observation (Fig. 4) that the potentially turbulent zones in the denser and more stable plasmas lay further out where the currents generate little power; moreover the energy released by the sawteeth (as measured by the radius of 
the magnetic surface $q=1$ ) is larger in the more stable of the two TFR discharges. Finally this hypothesis leads to an explanation of much of the ontology of sawtooth relaxations and plasma disruptions (Sec. V).

IV. COMPARTSON OF THEORETICAL AND EXPERTMENTAL ELECTRON HEAT TRANSPORT In order to derive the curves of anomalous electron thermal conductivity from experimental data, a number of physical assumptions have to be introduced. Identifying the different entries in the heat balance equation

$$
-\vec{\nabla}\left(\chi_{e}^{b_{N V}}+\vec{T}_{e}\right)=E J-P_{e i}-P_{\operatorname{rad}}-\frac{3}{2} \frac{\partial \mathrm{P}}{\partial t}
$$

requires for instance knowledge of the mechanisms of electron-ion energy transfer and of electrical resistivity. Both are usually assumed to be classical albeit the latter is sometimes corrected for electron trapping (44). Just as the hypothesis of classical energy exchange is infirmed in the theory of anomalous transport induced by drift wave turbulence (see Eq. (14)), there is no compeling reason for which the ohmic power which is slowly stored in the poloidal magnetic field should be absorbed exclusively by the electrons when released in bursts during the sawteeth relaxations (45). To account at least qualitatively for the possible deficiencies in the identification of the losses through the ion channel, we shall thus distinguish between actual and classical energy transfer. Tdentifying Eqs. (14) and (17) then yields

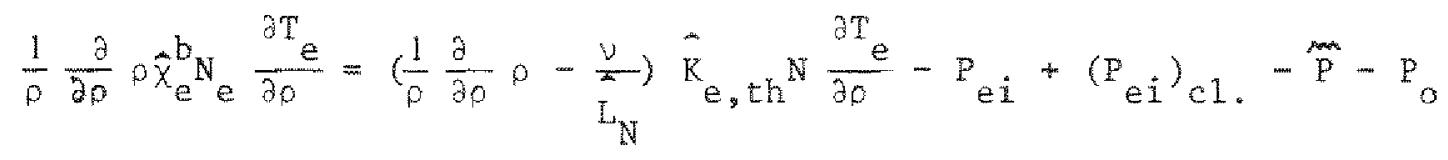

where the lengths have been nornalized to the plasma radius and 


$$
a^{2} \hat{k}_{e, t h} \equiv \frac{5<\alpha\rangle}{3}\left(\frac{m_{e}}{m_{i}}\right)^{1 / 2} \frac{\tau^{1 / 2}}{k_{r c_{i}^{5}}^{5} a^{5}} v^{t-1} b_{p}\left(v^{* 1 / 2}\right) G_{1} \frac{a_{s}^{2} c_{s}}{\mid L_{s} T}
$$

The solution of the differential equation (18) is readily obtained in the form

$$
\begin{aligned}
& \rho N^{-\nu} g \hat{K}_{e, t h}-\rho N^{-\nu} g \hat{x}_{e}^{b}-\left(\rho N^{-\nu} g \hat{x}_{e}^{b}\right)_{1}+\int_{\rho_{1}}^{\rho} d \rho^{\prime} \rho^{n} N^{-v}\left[\frac{g \hat{x}_{e}}{I_{N}}\right. \\
& \left.-\mathrm{P}_{e i}+\left(\mathrm{P}_{e i}\right)_{\mathrm{cl}}-\mathrm{P}^{\mathrm{p}}-\mathrm{P}_{\mathrm{o}}\right]
\end{aligned}
$$

where the flux $g \hat{x}_{e}^{b}=-P_{e} \hat{x}_{e}^{b_{T}}{ }_{T}^{-1}$ has been introduced $\left(\hat{L}_{T}^{-1}=d \ln T_{e} / d g<0\right) ; \rho_{1}$ is the normalized radius corresponding to the onset of the dissipative trapped electron instability. Electron-ion energy transfer induced by drift waves turns out to be negligible $(\nu \ll t)$. Assuming that $\mathrm{P}_{\mathrm{ei}}-\left(\mathrm{P}_{\mathrm{ei}}\right)_{\mathrm{cl}}=-\zeta \mathrm{c}$, $\mathrm{i} . \mathrm{e}$. a fraction $\zeta$ of the energy released by the sawteeth $(-\vec{P})$ goes into the ion channe1, and

$$
\int_{\rho_{1}}^{\rho} d \rho^{\prime} \rho^{\prime} \underline{p}=-\left(\rho g \hat{x}_{e}\right) \rho_{1}\left\{1-\exp \left[-\theta\left(\rho-\rho_{1}\right)^{2}\right]\right\}=-\left(\rho g \hat{x}_{e}^{b} \rho_{1}[1-h(\rho)]\right.
$$

Eq. (19) finally reduces to the form

$$
\begin{aligned}
\left(\rho g \hat{\mathrm{K}}_{e}\right)_{t h} & =\rho g \hat{x}_{e}^{b}-\left(\rho g \hat{x}_{e}^{b}\right)_{1}\{1-(1-\zeta)[1-h(\rho)]\} \\
& =\left(\rho g \hat{K}_{e}\right)_{\exp }^{*}
\end{aligned}
$$

if $\mathrm{P}_{0}=0, p_{1} \leq \rho \leq p_{2}$. The function in the curly brackets is unity at $p=p_{1}$ and decreases steadily thereafter; in first approximation this behaviour agrees qualitatively with the experimental curves of $\mathrm{P}$ (21). Rather arbitrarily we have also assumed that $h\left(1.5 p_{1}\right)=0.4$. 
As anticipated in Sec. IIr, the values of the parameter which are required to obtain a rough fitting of both sides of Eq. (19) are different for the three discharges: $\mu \cong 1$ (TFR moderate density), $\mu \cong 2$ (TFR high density), and $\mu \equiv 2.7$ (FT) $(\lambda=2$ is assumed throughout in the numerical calculations). It is not clear whether these discrepencies can be accomodated within the "error bars" of the theoretical linear growth rate (to which the transport is extremely sensitive, see below), and of some convenient simplifications such as the uniform distribution of impurities, or whether a more subtle modification of attitude is requested in relation to the heat balance equation. If triggered by the energy flows released by the sawtooth relaxations of the core, the instability of the trapped electron mode and the anomalous electron heat fluxes could indeed be pulsed especially in high density plasmas. The stability properties of the time average profiles would then not be fully relevant.

Figures (5) and (6) further suggest that the trapped electron mode must mostlikely be replaced by some other transport mechanism near the plasma edge where the collisionality parameter becomes quite large. Whether an appreciable fraction $(c)$ of the energy released by the relaxations is conducted away through the ion channel or not does not modify significantly the conclusions. The short wavelength branch of the fluctuation spectrum shown in Fig. 7 is predicted theoretically (7-9) but is not observable with present detection systems. It is found to account for almost all of the drift wave contribution to the anomalous electron heat flux ( $90 \%$ ); it does not bring however any significant contribution to the density fluctuation level. The theory thus permits to resolve the contradiction which appears when one notes, on the one hand, that plasma profiles are almost always close to marginal stability (18) - this indicates that the themal conductivity of the dissipative trapped mode is high - and, on the other hand, that the transport estimated on the basis of the observed spectrum (the long wavelength branch) is insufficient 
to account for the missing power in the energy balance equation. In this respect, we note that the factor by default ( $=3$ ) quoted by the TrR team (15) is largely underestimated because, as they have emphasized, the radial localization of the trapped electron response was not taken into account in their calculation. The actual missing factor should be of the order of

$3\left(\mathrm{x}_{\mathrm{c}} / \Delta\right)_{2} \simeq \mathrm{k}_{\theta} a_{i} \sqrt{2} \mathrm{qR} / \tau \mathrm{L}_{\mathrm{n}} \simeq 10$ for $\rho=0.5=\mathrm{k}_{\theta} \mathrm{a}_{i}$.

In order to check that the trapped electron mode has potentially a good thermal conductance, we have multiplied the destabilizing term of Eq. (7) by $1.5 ; \mathrm{K}_{\mathrm{e}}$ given by Eq. (18b) increased correspondingly by a factor in excess of 10. This result not only gives a theoretical basis for the observed relaxation of the profiles to a nearly marginal state, but it also implies that the turbulent transport of the transition layer cannot be described by scaling laws. Attempts to do so in the past $(11,19)$ have effectively led to apparently unsatisfactory results. The marginal stability condition rather yields a relation between the temperature, the density, their gradients, etc. ... which, when combined with $\mathrm{Ohm}^{\prime} \mathrm{s}$ law, has some resemblance to the Murakami high density limit $(20,21)$. This has prompted us to search for an interpetation of this limit in the framework of the present transport model. We note that a density increase stabilizes the trapped electron mode and thus clamps the radial energy outflux. When the core is no longer able to put up with the increasing pressure, energy dumping has to proceed through sudden readjustments of the profiles. This question is further analysed in the next Section.

The turbulenece levels are given by the equation cf.Eq.

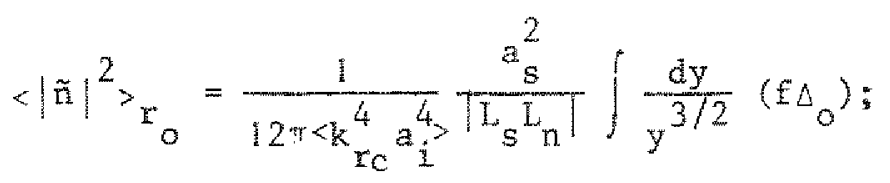

they reach their maximum values $\left\langle|\tilde{n}|^{2} \mathrm{th}=3.210^{-6}\right.$ and $1.610^{-6}$ at the normalized radii $\rho=0.5$ and $\rho=0.65$ in the moderate density and the high 
density discharges of TFR tespectively: These are somewhat lower than the measured values at $\rho=0.5$, respectively \langle| tit $\left.\right|^{2} \exp \equiv 1.610^{-5}$ and $410^{-6}$. The discrepencies are clearly not significatively given the experimental uncertainties and the possible optimization and improvenents of the theory. We have not drawn the curves of the transport coefficient for the FT discharge since we have no information on the relaxations (the machine was actually operating at a fairly large $\sigma_{a}=4.9$ ). We merely note that with $\mu=2.7$ and $\lambda=2$, we obtain $\left.\rho_{1}=0.25, \rho_{2}=0.78,<|\tilde{\mathfrak{n}}|^{2}\right\rangle_{\max }=2.840^{-6}$ at $p=0.6$, and $\left(K_{e} N\right)$ th $=3.410^{17} \mathrm{~cm}^{-1} \mathrm{sec}^{-1} \operatorname{compared}$ to $\left(\mathrm{K}_{\mathrm{e}} \mathrm{N}\right)_{\mathrm{exp}} \mathrm{m}^{3} 10^{17}$ $\mathrm{cm}^{-1} \mathrm{sec}^{-1}$ at the radius $0=0.5$ where the theoretical value is maximum.

V. ORIGIN OF THE SAWTOOTH RELAXATIONS AND OF THE MAJOR DISRUPTIONS

In contrast to binary collisions, the mechanisms of anomalous transport are triggered and controlled by the flows themselves. Because of this autom regulating process, a discharge supplied continuously with power can develop a cycic patern.

Two scenarios can effectively be forecast.

\section{A. First Scenario}

The heat flux carried away through the internediate layer by drift waves and neoclassical processes catches up with the ohmic power deposition before the conditions for the onset of relaxations are reached in the core. The transport will be fully laminar; the energy content and the pressure profile will settle in a stationary state. The viabilty of this scheme requires that binary collisions and pexhaps a collisionless microinstability [see Fig. 2 and the discussion following Eq. (16b)] provide the transport in the core itselt. It probably applies to the high density Alcator and ET dischaxges where sawtooth oscillations are not seen or have a negligible amplitude $(46,47)$ 
B. Second Scenario

The conditions for the onset of relaxations are reached in the core before the heat flux across the intermediate layer can balance the ohmic power deposition. The excess energy trapped in the core will be released in the form of bursts when the latter are able to raise the turbulent transport in the surrounding layer to a sufficient level. This can be achieved (Order of magnitude estimates are given in Appendix B) since the heat pulse decreases the collisionality along its path and hence increases the trapped electron contribution to the growth rate [cf. Eq. (7)]. The amplitude of these relaxations will naturally depend on how deeply the energy is trapped in the core. Eventually a major catastrophy will arise: the gross plasma disruption.

The explanation of plasma relaxations and disruptions that we put here forward is consistent with many of the experimental information gathered on these crucial issues:

(i) the amplitude and the period of the sabtooth relaxations increase with increasing plasma density (21). The latter indeed reduces the destabilizing role of the trapped electrons [Eq. (7)] and hence improves the thermal insulation of the core.

(ii) a gross plasma disruption occurs at a given high density limit $(20,21)$. Relating the latter to the stability of the trapped electron mode across the predisrupted discharge yields indeed the following density limit (see Appendix C)

$$
N_{0}=610^{16} \frac{a^{1 / 2} \mathrm{~T}_{e, 0}^{2}}{R^{3 / 2} A_{i}^{1 / 2}} \frac{\mu F\left(a / q_{a} R\right)}{1+Z_{e f f}}
$$

where $A_{i}$ is the atomic mass. The functional $F$ depends on the assumed density and temperature profiles and on the assumed drift stability limit; $F(0.1)=1$ 
with the hypothesis introduced in the Appendix. With the helr of onm's law, Eq. (22a) also writes

$$
N_{0}=4.210^{10} \frac{a^{1 / 2}}{R^{1 / 6} A_{i}^{1 / 2}}\left(\frac{B_{t} Z_{e f f}}{q_{o} R V}\right)^{4 / 3} \frac{\mu F\left(a / q_{a} R\right)}{1+Z_{e f f}}
$$

which clearly reminds of the Murakami limit (20). In Eqs. (22a) and (22b), $\left[T_{e}\right]=\operatorname{kev},[\mathrm{V}]=$ volt, $[B]=$ Gauss, and $[\mathrm{L}]=\mathrm{cm}$. Numerically, $\mathrm{N}_{\mathrm{o}}=310^{14} \mathrm{~cm}^{-3}$ - if $Z=V=A_{i}=H=2, q_{a}=3, B_{E} / q_{0}=5 \cdot 10^{4}, a=20$ and $R=10^{2}$ - is fully satisfactory in view of TrR results $(21)$.

(iii)" The observations are consistent with the model of distuption in which ohmic heating is insufficient to prevent cooling of the plasma edge; this leads to contraction of the colum, a lowering of $q$ at the boundary and a steepening in the radial gradient of current density leading eventually to magnetohydrodynamic instability and discuption." (48) we argue here that the cooling of the plasma periphery is the consequence of the momentary clamping of the heat outflow from the core, We argue also that the MDD precursors excited by the shrinking of the current chamel are not the nost plausible cause for the disruptive transport although they may acknowledgedly help in unloading the core. Actually no MHD oscillations precede the disruptions triggered in Alcator by injecting gas above the density imit (46).

iv) the Murakami density limit has been passed with neutral beam heating (49). In our model, this result follows immediately from Ea. (22a) which also predicts that higher densities could be maintained by the thermonuclear heating.

v) the heat bursts from the core reach the plasma periphery within a very short time compared to the electron energy confinement time $(50,51)$. This is easily explained in view of the strongly nonlinear dependence of the anomalous heat conductivity with linear growth rate (see sec. IV), the latter being enhanced when the pulse passes by. 
The theory predicts furthermore that $\beta_{\theta}$, the ratio of the kinetic and poloidal magnetic pressures, should be bounded from above independently from the current - but not from the loop voltage. The theoretical limit is set by the disruptions as observed in the Pulsator experiment (52). With the help of Eq. (22a) and $\mathrm{Ohm}^{\prime} \mathrm{s}$ Iaw, there results indeed that

$$
\left(B_{p}\right)_{\operatorname{Max}}=\frac{f d \tau \mathrm{Nk}\left(\mathrm{T}_{\mathrm{e}}+\mathrm{T}_{\mathrm{i}}\right)}{\tau\left(\mathrm{B}_{\mathrm{p}}^{2} / 8 \pi\right)_{\mathrm{r}=\mathrm{a}}}=125 \frac{\mathrm{R}^{1 / 2}}{\mathrm{a}^{3 / 2} \mathrm{~A}_{i}^{1 / 2}}\left(\frac{\mathrm{eff}_{\mathrm{V}}}{\mathrm{V}}\right)^{2} \frac{\mathrm{F}^{*}\left(\mathrm{a} / \mathrm{q}_{\mathrm{a}} \mathrm{R}\right)}{1+\mathrm{Z}_{\mathrm{eff}}}
$$

where $F^{*}(0.1)=1$ only if one introduces the hypothesis of Appendix $C$ : in view of these the numerical coefficients in Eqs. (22) and (23) are merely representative. The units are those of Eqs. $(22 a, b)$. We have taken $\mathrm{N}_{i} \mathrm{~T}_{i}=\mathbb{N}_{e} \mathrm{~T}_{e}$.

We should like to add some precisions. When the heat flux is clamped in the transition layer, the core "overheats" and the periphery "overcools". Hence the Minov oscillations (53) and also the perturbations of the recycling processes (54). The $\mathrm{m}=1$ oscillations can lead in their nonlinear stage to a flattening (relaxation) of current density, safety factor, and temperature profiles inside the $q=1$ magnetic surface (55) but not to an unloading of the core. We have implicitely assumed that this flattening occurs only when the heat wave is able to make its way through the surrounding layer by exciting the trapped electron mode to a sufficient level. This hypothesis, which is reminescent of the physical principles of collisionless shock waves (56), is quite plausible from an energetic point of view since otherwise the reflection of the heat pulse on the $q=1$ surface would certainly tend to prevent the collapse of the profiles. The transition to the more dramatic najor distuptions could conceivably occur when the $q=1$ and the $q=2$ magnetic islands start to overlap as proposed by Stix (57). Even in this case Eqs. (22a) and (22b) for the density limit should be valid since the ultimate cause of magnetic braiding would be the quenching of the trapped electron mode. 
VI. CONCLUSIONS

Analysis of three discharges in TFR and in FT $(15,16)$ has confirmed

- that tokamak profiles are weakly (un-)stable to the trapped electron mode

(18): this proves that this instability plays a role in the transport;

- that toroidal effects are too weak to neutralize shear damping.

The theory of drift wave saturation via nonlinear ion Landau damping

presented separately (7-9) provides an explanation of

- the discordance between the anomalous heat conduction coefficients

estimated (a) from the equation of power balance and (b) on the basis

of the measured fluctuation spectrum (15),

- the relaxation of the profiles to a marginally (un-)stable state (18),

- the origin of the sawtooth relaxations and the gross plasta distuptions,

- the increase of the amplitude and period of the sawteeth with plasma density $(21)$,

- the high density limit in Ohmic plasmas $(20,21)$ and its overtaking with neutral beam heating (49) (or more generally with auxiliary heating),

- the contraction of the plasma column (48),

- the surprisingly rapid propagation to the plasma edge of the heat

pulses released by the relaxations of the core (50-51),

Erom first principles.

It finally implies that drift wave transport cannot be described by scaling laws.

It will be shown in another paper that the theory yields moreover realistic diffusion coefficients for high $z$ impurities and provides an explanation of the cleaning action of the sawteeth.

\section{ACKNOWLEDGEMENT}

We have benefited from many discussions with and constant encouragement Erom Prof. F. Waelbroeck. A useful conversation with Dr. K. Lackner is also acknowledged. 


\section{APPENDTX A}

Application of the Theory of Residues in the Calculation of the Sum in Eq. (12a)

Indeed (36)

$$
\begin{aligned}
\int_{-\infty}^{\infty} \frac{1[(t+p) u] J_{1}[(t-p) u]}{t^{2}-p^{2}} & =\frac{2 J_{3 / 2}(2 u p)}{(\pi u)^{1 / 2} p^{3 / 2}} \\
& =\frac{2}{\pi u p^{2}} \quad\left(\frac{\sin 2 u p}{2 u p}-\cos 2 u p\right)
\end{aligned}
$$

Consider the function

$$
f(z)=-\frac{1}{2}\left(\frac{\dot{i}}{2 u z}+1\right) \frac{\exp [i z(2 u-\pi)]}{z^{2} \sin \pi z}
$$

It vanishes exponentially on the contour $|z| \rightarrow \infty$ the sum of its residues in the complex plane is thus zero leading to

$$
\begin{aligned}
-\sum_{p \neq 0}\left(\frac{i}{2 u p}+1\right) \frac{\exp (2 i u p)}{p^{2}}= & \frac{4(u-\pi / 2)}{3 ! u}\left[(u-\pi / 2)^{2}-(\pi / 2)^{2}\right]+ \\
& +4\left[-\frac{(u-\pi / 2)^{2}}{2}+\frac{(\pi / 2)^{2}}{3 !}\right] \\
& =\sum_{p \neq 0} \frac{1}{p^{2}}\left(\frac{\sin 2 u p}{2 u p}-\cos 2 u p\right) .
\end{aligned}
$$

Integrating over the variable u yields

$$
\left\langle\phi_{\ell, m}^{*} \mid \sum_{p \neq 0} \phi_{\ell, m+p} S_{p}\right\rangle_{r_{0}}=(10 / 9) \pi^{2}\langle\alpha\rangle_{\theta} q^{-1}|\phi(0)|^{2}
$$

whereas, with the help of $(\mathrm{A}-1)$ where $\mathrm{p} \rightarrow 0$, 


$$
\left\langle\phi_{\ell, m}^{*} \mid \phi_{\ell, m} S_{0}^{>}=(8 / 9) \pi^{2}<\alpha\right\rangle_{\theta} q^{-1}\left\{\left.\phi(0)\right|^{2} .\right.
$$

The contribution from the pfo terms should be taken into consideration only if $\Delta^{2} t^{2} / 4 x_{t}^{2} \leq \pi / 2$ where $t \pi / 2=$ first null of the Bessel function. The transition region thus occurs for $\Delta^{2} / x_{t}^{2} \sim 1$. Note that the result quoted in the text for $A / x_{t} \ll I$ can also be recovered with the help of equation (23) of Reference $(22)$.

\section{APRENDIX B}

Effect of the Heat Pulse Released from the Core on the Stability of Drift Waves in the Transition Layer

We consider profiles of the form $N=N_{0}\left(1-\rho^{2}\right), T=T_{0}\left(1-\rho^{2}\right)^{2}$ with $\rho=I / a$. Assuming that the relaxation of the core takes place inside the rational surface of radius $r_{1}=a p_{1}$, and concerns only the temperature, the released energy is

$$
\Delta E=2 \pi a^{2} \int_{0}^{1} \rho d \rho N\left(T-T_{1}\right)=\frac{\pi a^{2}}{4} N_{0} T_{0}\left[1-\left(1-p_{1}^{2}\right)^{2}\right]^{2} .
$$

This corresponds to a fraction $n=\left[1-\left(1-p_{1}^{2}\right)^{2}\right] /\left[1+\left(1-p_{1}^{2}\right)^{2}\right]$ of the energy content of the core. After a given lapse of time the heat pulse will be confined within the magnetic surfaces $r_{1}$ and $r_{2}$ where it will represent a fraction

$$
\frac{\Delta E}{E_{1,2}}=\frac{\left[1-\left(1-\rho_{1}^{2}\right)^{2}\right]^{2}}{\left(1-\rho_{1}^{2}\right)^{4}-\left(1-\rho_{2}^{2}\right)^{4}}
$$

of the unperturbed energy content. The destabilizing trapped electron term in $\mathrm{Eq}$. (7) being proportional to $\mathrm{T}^{2}$ increases as $\left(1+\Delta E / \mathrm{E}_{1,2}\right)^{2}$. If the amplitude of the pulse remains constant as it propagates, then $p_{2}=\sqrt{2} p_{1}$. However, if the transport is still not sufficient the pulse will decelerate with the consequence that $p_{2}<\sqrt{2} p_{1}$. 
Numerically, if $\rho_{1}=0.4, \eta=0.175$ is of the order of the central temperature relaxation in TFR (21). If $\rho_{2}=\sqrt{2} \rho_{1}$, the average increase of temperature in the layer $\rho_{1}<\rho_{2}$ is

$$
\left(\frac{\Delta \mathrm{T}}{\mathrm{T}}\right)_{1,2}=\frac{\Delta \mathrm{E}}{\mathrm{E}_{1,2}}=0.30
$$

the destabilizing trapped electron term is thus multiplied by 1.7 owing to decreasing collisionality. This shows that the heat pulse is capabie from an energetic point of view of destabilizing the turbulence to sustain its own transport. We should like also to note that the observed decay time of the sawteeth $\left(\sim 10^{-4} \sec \right)(13,14)$ is sufficient for the drift waves to reach their saturated suprathermal level $\left(\gamma \sim k_{\theta} a_{s} c_{s} / L_{s} \sim 10^{5} \mathrm{sec}^{-1}\right)$.

\section{APPENDIX C}

Estimation of the High Density Limit Arising from the Quenching of Drift Wave Transport

We assume the same density and temperature profiles as in Appendix B. The amplitude of the heat pulse necessary to drive the turbulence sustaining the transport depends from the growth rate in the unperturbed state (i.e. the state between the relaxations). We postulate rather arbitrarily - but we believe the results are not very sensitive to the uncertainty which is thus introduced - that the maximum relaxations that the discharge can withstand without distupting occur when the trapped electron mode is at most marginally unstable between two relaxations. The equations $\tilde{\gamma}=0, \partial \tilde{\gamma} / \partial y=0$, and $\partial \tilde{\gamma} / \partial \rho=0, \dot{i}, e$.

$$
\exp \left(-\lambda \Delta / x_{t}\right)=1-(\mu c)^{-1} \frac{1+y}{\sqrt{y}}
$$




$$
\begin{aligned}
& =\frac{1-y}{1-y+\lambda \Delta / x_{t}} \\
& =\frac{\partial \ln (\mu c) / \partial \rho}{\partial \ln (\mu c) / \partial \rho-\lambda \partial\left(\Delta / x_{t}\right) / \partial \rho},
\end{aligned}
$$

then yield a relation between the quantities

$$
\begin{aligned}
& C=u\langle\alpha\rangle_{\theta}\left(\frac{\pi}{2}\right)^{7 / 2} \frac{3 c_{s, 0}}{\left(1+z^{-1}\right) v_{e i, 0} R^{1 / 2} a^{1 / 2} q_{a}} \\
& A=\left(\frac{2 a}{R q_{a}}\right)^{1 / 2}
\end{aligned}
$$

where the indices " $O$ " and "a" correspond to the radi $r=0$ and $r=a$. With these definitions,

$$
\mu c=c \frac{\rho^{3 / 2}\left(1-\rho^{2}\right)}{3-4 \rho^{2}+3 / 2 \rho^{4}}
$$

and

$$
\frac{\Delta}{x_{t}}=A \frac{\left(1-p^{2}\right)^{1 / 2}\left(1-3 / 2 p^{2}+p^{4}-1 / 4 p^{6}\right)}{\left(3-4 p^{2}+3 / 2 p^{4}\right)^{1 / 2} p^{3 / 2}}\left(\frac{1+y}{y}\right)^{1 / 2}
$$

Table I gives the solutions of Eqs. $(C-1)$ through $(C-3)$ as function of the parameter A. Note that

$$
\begin{aligned}
\mathrm{CA}^{2} & =2210^{16} \frac{a^{1 / 2} \mathrm{~T}_{e, o}^{2}}{\mathrm{R}^{3 / 2} \mathrm{~A}_{\mathrm{i}}^{1 / 2} \mathrm{~N}_{\mathrm{o}}}\left(1+\mathrm{z}_{\mathrm{eff}}\right)^{-1} \mathrm{H} \\
& =3.6 \mathrm{~F}^{-1}\left(\mathrm{a} / \mathrm{q}_{\mathrm{a}} \mathrm{R}\right)
\end{aligned}
$$

with the definition of Sec. V, i.e. $F(0,1)=1,0^{*}$ is the radius where the mode is marginally unstable, and $y^{*}=k_{\theta}^{2} a_{s}^{2}$ yields the correspondirg mode number. 


$\begin{array}{llll}\text { a/Rqa } & F & \rho^{*} & y^{*} \\ 0.150 & 0.75 & 0.656 & 0.47 \\ 0.125 & 0.85 & 0.647 & 0.45 \\ 0.100 & 1 & 0.635 & 0.44 \\ 0.075 & 1.23 & 0.621 & 0.41 \\ 0.050 & 1.64 & 0.6 & 0.38 \\ 0.025 & 2.63 & 0.564 & 0.34\end{array}$

TABLE I 
FIGURE CAPTIONS

Fig. 1 Experimental profiles of density and temperatures from Refs. (15) and (16). Calculated profile of safety factor assuming $Z_{\text {eff }}=$ constant and classical resistivity. (a) TFR Moderate Density; (b) TFR high density; (c) FT.

Fig. 2 The range of poloidal mode number for which shear damping is annihilated lies between the two limiting curves (- TFR moderate density; -.-.- TER high density; -....- FT)

Fig. 3 Comparison between electron detrapping collision frequency and (a) inertia, (b) average magnetic drift. (Same convention as in Fig, 2).

Fig. 4 Limit of the unstable layer as function of the paraneters $\lambda$ and $\mu$. (Same convention as in Fig. 2)

Fig. 5 Maximum growth rates obtained from $\mathrm{Eq}$. (7) with $\lambda=2$ and $\mu$ as specified in the text. The corresponding value $y$ (Max) is given. The conventions are as in Fig. 2 .

Fig. 6 Heat conduction coefficient obtained (a) from theory and (b) from experiment (i.e. power balance considerations). The conventions are as in Fig. 2 . 


\section{REEERENCES}

1 Kadomtsev, B.B., Pogutse, O.P., Reviews of Plasma Physics VoI. 5 , Consultant Bureau, New York (1970) 249. Taylor, J.B., in Plasma Physics and Controlled Nuclear Fusion Research (Proc, 6th Int. Conf. Berchtesgaden, 1976) Vol. II, IAEA, Vienna (1977) 323.

3 Chen, L., Guzdar, P.N., Hsu, J.Y., Kaw, P.K., Oberman, C., White, R., Nuc1. Fusion 19 (1979) 373.

4 Hassetberg, G., Rogister, A., Plasma Phys. 22 (1980) 805.

5 Chen L., Cheng, C.L., Phys. Fluids 23 (1980) 2242; Cheng C.Z., Chen L., Nuc1. Eusion (1981) 403.

6 Choi, D.I., Horton, W., Phys. Fluids 23 (1980) 356.

7 Rogister, A., Hasselberg, G., Fusion Energy - 1981 (Proc. Spring College on Fusion Energy, Trieste, 1981) TAFA, Vienna (1982) 51.

8 Rogister, A., Hasselberg, G., Phys. Rev. Lett. 48 (1982) 249.

9 Rogister, A., Hasselberg, G., to appear in Phys. Fluids.

10 Pearlstein, L.D., Berk, H. L., Phys, Rev. Lett. 23 (1969) 220.

11 Hasselberg, G., Rogister, A., E1-Nadi A., Phys. Fluids 20 (1977) 982; Horton, W., Phys. Rev. Lett. 37 (1976) 1269. The two papers consider the same nonlinear process, namely nonlinear ion diamagnetic drifts, The shielding associated with the polarization is neglected in the second paper.

12 Hirshman, S.P., Molvig, K, Phys. Rev. Lett. 42 (1979) 648.

13 Von Goeler, S., Stodiek, W., Sauthoff, N., Phys. Rev. Lett. 33 (1974) 1201; von Goeler, S., in Controlled Fusion and Plasma Physics (Proc. 7th European Conf. Lausanne, 1975) VoI. II (1975) 71 .

14 Equipe TFR, in Plasma Physics and Controlled Nuclear Fusion Research (Proc.6th Int. Conf. Berchtesgaden, 1976) Vol. T, IAEA, Vienna (1977) 279 and 35. 
Equipe TrR, in Plasma Physics and Controlied Nuclear Fusion Research (Proc, 8th Int. Conf. Brussels, 1980) Vol. I, IAEA, Vienna (1981) 425. Report guR-CEA-FC-1113 (1981), unpublished. Bartiromo, R., Brusati, M., Buratti, P., Cilloco, F., De Marco, F., Gasparotto, M., Giannella, R., Mazzitelli, G., Pieroni, F., Santini, F., Scovenna, M., Tucillo, A., Tudisco, O., Zanza, V., in Plasma Physics and Controlled Nuclear Fusion Research (Proc. 8th Int. Conf. Brussels, 1980) Vol. I, IAEA, Vienna (1981) 43.

17 It is actually difficult to ascertain that the modes are definitely unstable given the inaccuracies inherent both to the theory and to the experiments.

18 Manheimer, W.M., Antonsen, T.M., Phys. Fluids 22 (1979) 957.

19 Kadomtsev, B.B., Pogutse, O.P., in Plasma Physics and Controlled Nuclear Fusion Research (Proc.7th Int. Conf. Innsbruck, 1978) Vo1. 1, IAEA, Vienna (1979) 649.

20 Murakami, M., Callen, J.D., Berry, L.A., Nuc1. Fus. 16 (1976) 347.

21 Equipe T.F.R., Nucl. Fus. 20 (1980) 1227.

22 Rogister, A., Hasselberg, G., Phys. Fluids 22 (1979) 2382 (Sections V and ITI).

23 Rewoldt, G., Tang, W.M., Frieman, E.A., Phys. Fluids 21 (1978) 1513.

24 Braginskil, S.I., in Reviews of Plasma Physics Vol. T, Consultant Bureau, New York (1965) 215.

25 Bogoliubov, N.N., Mitropolsky, Y.A., Asymptotic Methods in the Theory of Nonlinear Oscillations, Hindustan Fublishing Corpn., Delhí (1961).

26 The concept of zero-damped modes is defined by Stix, T.H., The theory of Plasma Waves, McGraw-Hi11, New York (1962) 213.

27 Catto, P.J., Tsang, K.T., Phys. Fluids 21 (1978) 1381.

28 Single-mode marginal stability may be achieved either through relaxation in physical space or in velocity space. The one-point theory of re- 
ference 12 falls in the latter category.

29 Sagdeev, R.Z., Galeev, A.A., Nonlinear P1asma Theory, Benjamin, New York (1969) 89.

30 Rogister, A., Phys. Rev. Lett. 34 (1975) 80; Rogister, A., hasselberg, G, , Phys. Fluids $19(1976) 108$.

31 Chen, L., Berger, R.L., Lominadze, J.G., Rosenbluth, M.N., Rutherford, R.H., Phys. Rev. Lett. 39 (1977) 754 .

32 Mazzucato, E., Phys. Rev, Lett. 36 (1976) 792; Goldston, R.J., Mazzucato, E., Slusher, S., Surko, C.M., in Plasma Physics and Controlled Nuclear Fusion Research (Proc.6th Int. Conf. Berchtesgaden, 1976) Vo1. I, IAEA, Vienna (1977) 371.

33 TFR Group, in Controlled Fusion and Plasma Physics (Proc. 8th Eur. Conf. Prague, 1977) Vol. I, Institute of Plasma Physics, Prague (1978) 2.

34 Mazzucato, E., Phys. Rev. Lett. 48 (1982) 1828.

35 Slusher, R.E., Surko, C.M.; Phys. Rev. Lett. 40 (1978) 400.

36 Semet, A., Hase, A., Peebles, W.A., Luhman Jr., N.C., Zweben, S., Phys. Rev. Lett. 45 (1980) 445.

37 Obukhoff, A.M., C.R. Acad. Sci. URSS 32 (1941) 19; see also the review paper by Lin, C.C., Reid, W.H., Handbuch der Physik, (2nd edition, J. Sprinze, Beriin) (1964) 438.

38 Watson, G.N., Theory of Bessel Functions, University Press, Cambridge (1958) 422.

39 Fried, B.D., Conte, S.D., The Plasma Dispersion Function, Academic Press, New York (1961).

40 Ross, D.W., Mahajan, S.M., Phys. Rev. Lett. 40 (1978) 324; Tsang, K.T., Catto, P.J., Whiston, J.C., Snith, J., Phys. REv. Lett. 40 (1978) 327; Chen, L., Guzdan, P.N., White, R.B., Kaw, F.K., Oberman, C., Phys. Rev. Lett. 41 (1978) 649 . 
41 Cordey, J.G., Jones, E.M., Start, D.F.H., Plasma Phys. 21 (1979) 725; Rogister, A., Hasselberg, G., in Plasma Physics and Controlled Nuciear Fusion Research (Proc. 7th Int. Conf. Imsbruck, 1978) Vol. I, IAEA, Vienna (1979) 809.

42 Horton, W. Jr., Ross, D.W., Tang, W.M., Berk, H.L., Frieman, E.A., La Quey, R.E., Lovelace, R.V., Mahajan, S.M., Rosenbluth, M.N., Rutherford, P.H., in Plasma Physics and Controlled Nuclear Fusion Reseatch (Proc.5th Int. Conf. Tokyo, 1974) Vol. I, IAEA, Vienna (1975) 541.

43 Adan, J.C., Laval, G., Pellat, R., Nucl. Fusion 13 (1973) 47; Adan, J.C., Tang, W.M., Rutherford, P.H., Phys. Fluids 19 (1976) 561 ; Ross, D.W., Tang, W.M., Adam, J.C., Phys. Fluids 20 (1977) 613; 
Coppi, B., Rewoldt, G., Phys. Lett. A54 (1975) 301 and in Advances in Plasma Physics, Vol. 6, edited by A. Simon and W.B. Thompson, Interscience, New York (1976) 426.

44 Winsor, N.K., Hui, B.H., Coppi, B., in Theory of Magnetically Confined Plasmas (Proc. of the Course Varenna, 1977) Pergamon Press, Oxford (1979) 71.

45 Samain, A., Fusion Energy - 1981 (Proc. Spring College on Fusion Energy, Trieste, 1981) IAEA, Vienna (1982) 393.

46 Apgar, E., Coppi, B., Gondhalekar, A., Helava, H., Kom, D., Martin, F., Montgomery, B., Pappas, R., Parker, R., Overskei, D., in Plasma Physics and Controlled Nuclear Fusion Research (Proc. 6th Int. Conf. Berchtesgaden, 1976) Vol. I, IAEA, Viema (1977) 247.

47 Alladio, F., Bardott, G., Bartiromo, R., Buceti, G., Buratti, P., Crisanti, F., De Angelis, A, De Marco, F., de Pretis, M., Gasparotto, M., Giannella, R., Grolli, M., Maddaluno, G., Mazzitelli, G., Pieroni, L., Righetti, G.B., Santini, F., Segre, S.E., Tanga, A., Tuccillo, A., Tudisco, O., Zanza, V., Nucl. Fusion 22 (1982) 479. Bickerton, R.J., in Theory of Magneticaily Confined Plasmas (Proc. of the Course, Varenna, 1977) Pergamon Press (1979) 433. Axon, K.B., Clark, W.H.M., Cordey, J.G., Cox, M., Fielding, S.J., Gill, R.D., Hugil1, J., Lomas, P., Paul, J.M.W., Powel1, B.A., Prentice, R., Start, D.F.H., Sumers, D.D.R., Thomas, P., in Plasma Physics and Controlled Nuclear Fusion Research (Froc. 8th Int. Conf. Brussels, 1980) Vol. I, IAEA, Vienna (1981) 413.

50 Marty, D., private cormunication.

51 Lackner, K., private commuication.

52 Meise1, D., Klüber, O., Cannici, B., Engelhardt, W., Fussman, G., Gernhardt, J., Glock, E., Karger, F,, Lisitano, G, McCormick, K., Mayer, H.M., Morandi, P., Sesnic, S., Wagner, F., in Plasma Physics 
and Controlled Nuclear Fusion Research (Proc. 6th Int. Conf. Berchtesgaden, 1976) Vol. I, LAEA, Vienna (1977) 259.

53 Mirnov, S.V., Semenov, T.B., in Plasma physics and Controlled Nuclear Fusion Research (proc. 4th Int. Conf. Madison, 1971) Vol. II, IAEA, Vienna (1971) 401.

54 Enge1hardt, W., Kliber, O., Meise1, D., Murmann, H., Sesnic, S., Fussm mann, G., Glock, E., Gottardi, N., Karger, F., Lisitano, G., Mayer, H.M., Wagner, F., in Plasma Physies and Controlled Nuclear Fusion Research (Proc. 7th Int. Conf. Innsbruck, 1978) Vo1. I, IAEA, Vienna (1979) 123.

55 Kadomtsev, B.B., Fiz. Plazmy (Sov.J. Plasma Phys.) I (1975) 710.

56 Paul, J.M.W., Doughney, C.C., Holmes, L.S., Nature 223 (1969) 822; Bogen, P., Dietz, K.J., Dippe1, K.H., Hintz, E., Höthker, K., Siemsen, F., Zeyer, G., in Plasma Physics and Controlled Nuclear Fusion Research (Proc. 4th Tnt. Conf. Madison, 1971) Vol. III, IAEA, Vienna (1971) 277.

57 Stix, T.H., Phys. Rev. Lett. 36 (1976) 521. 


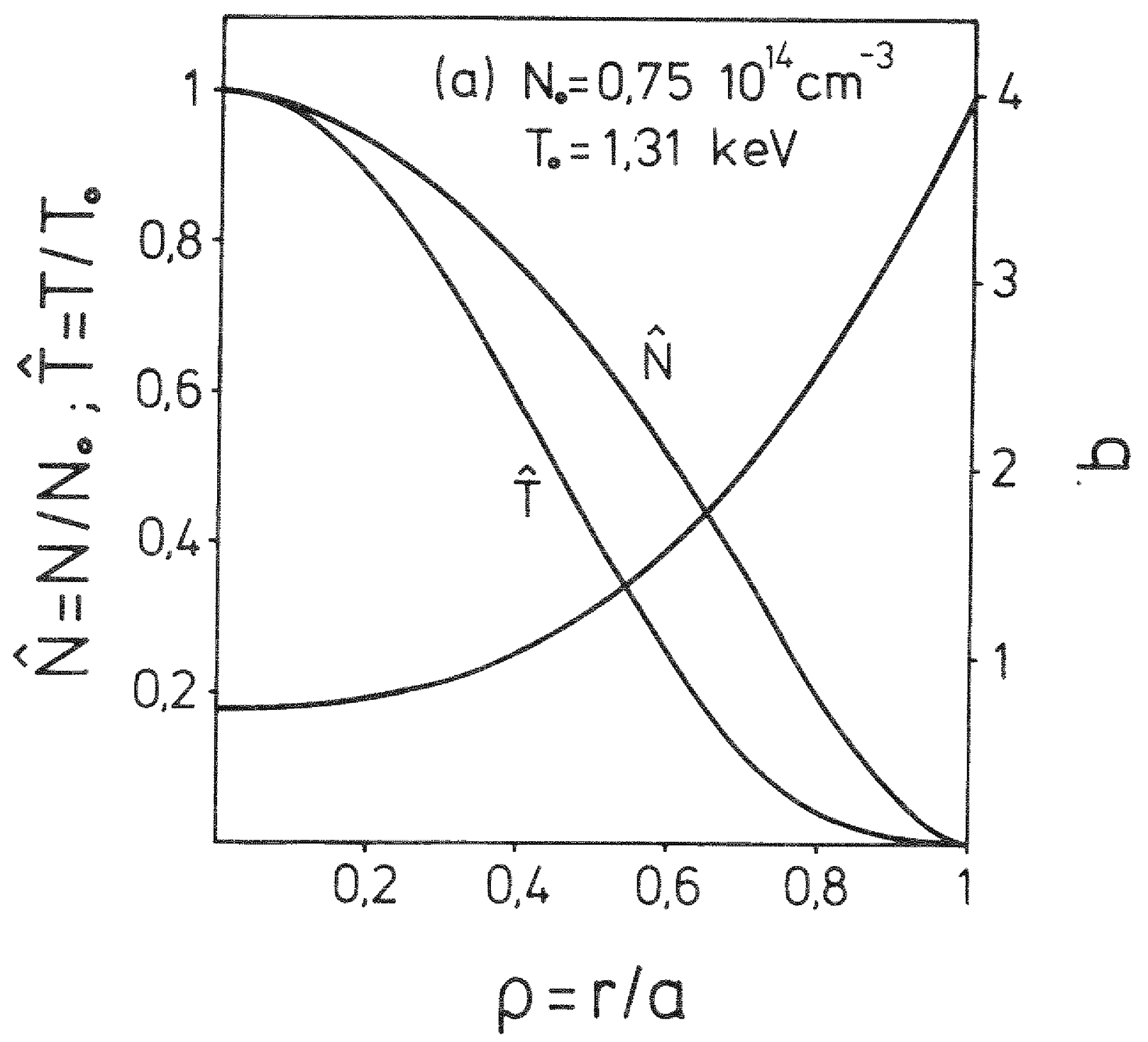




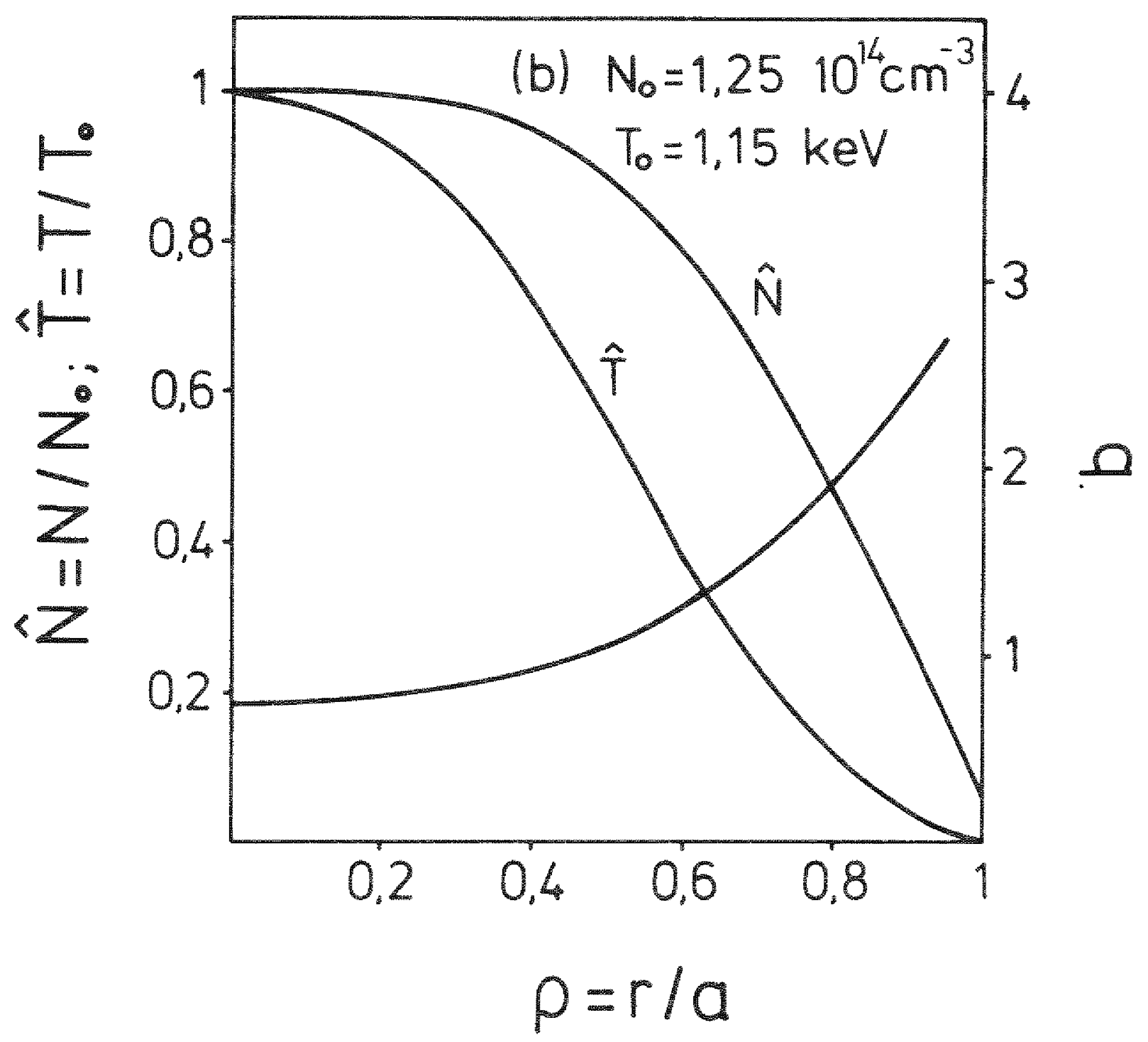




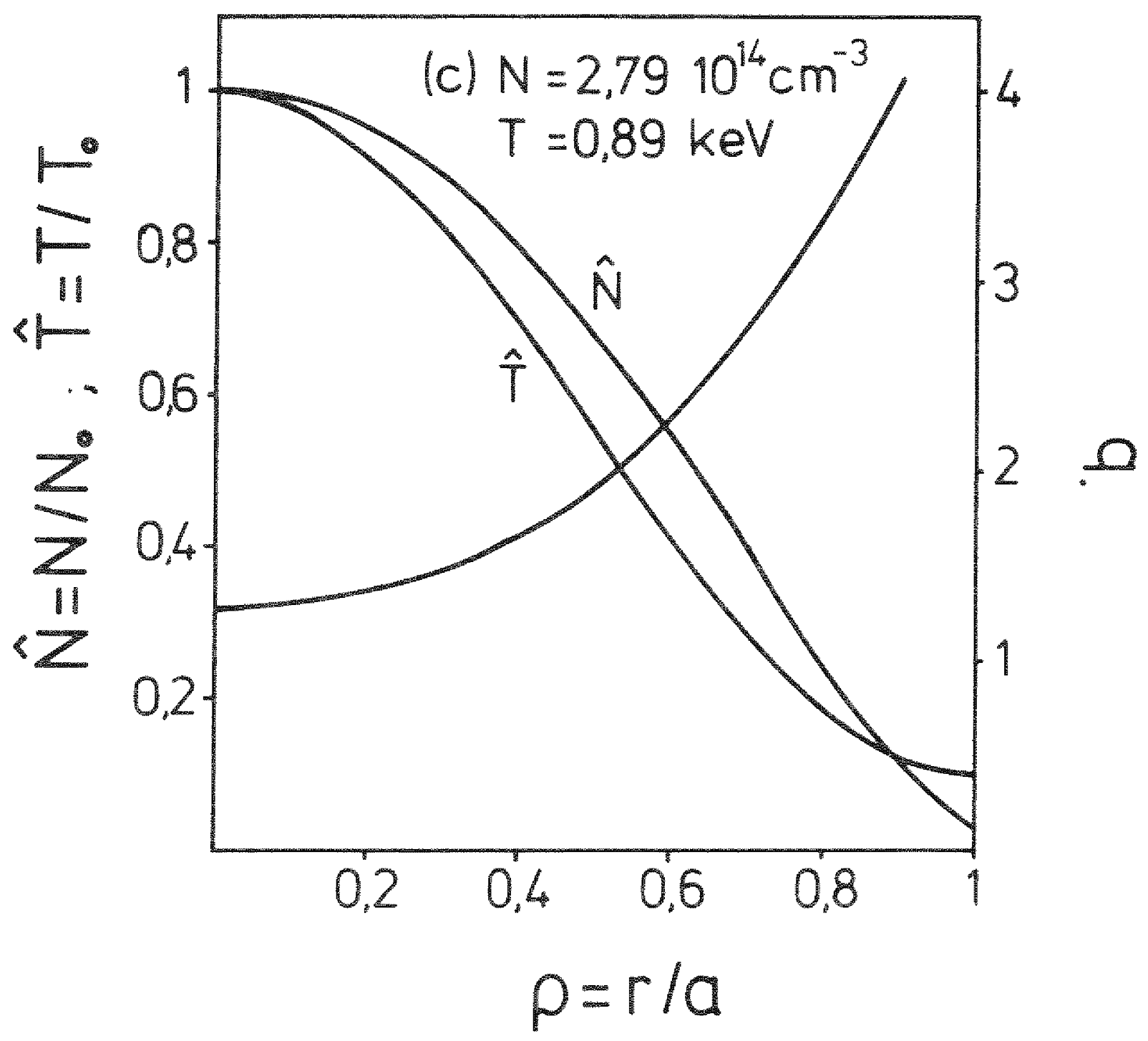




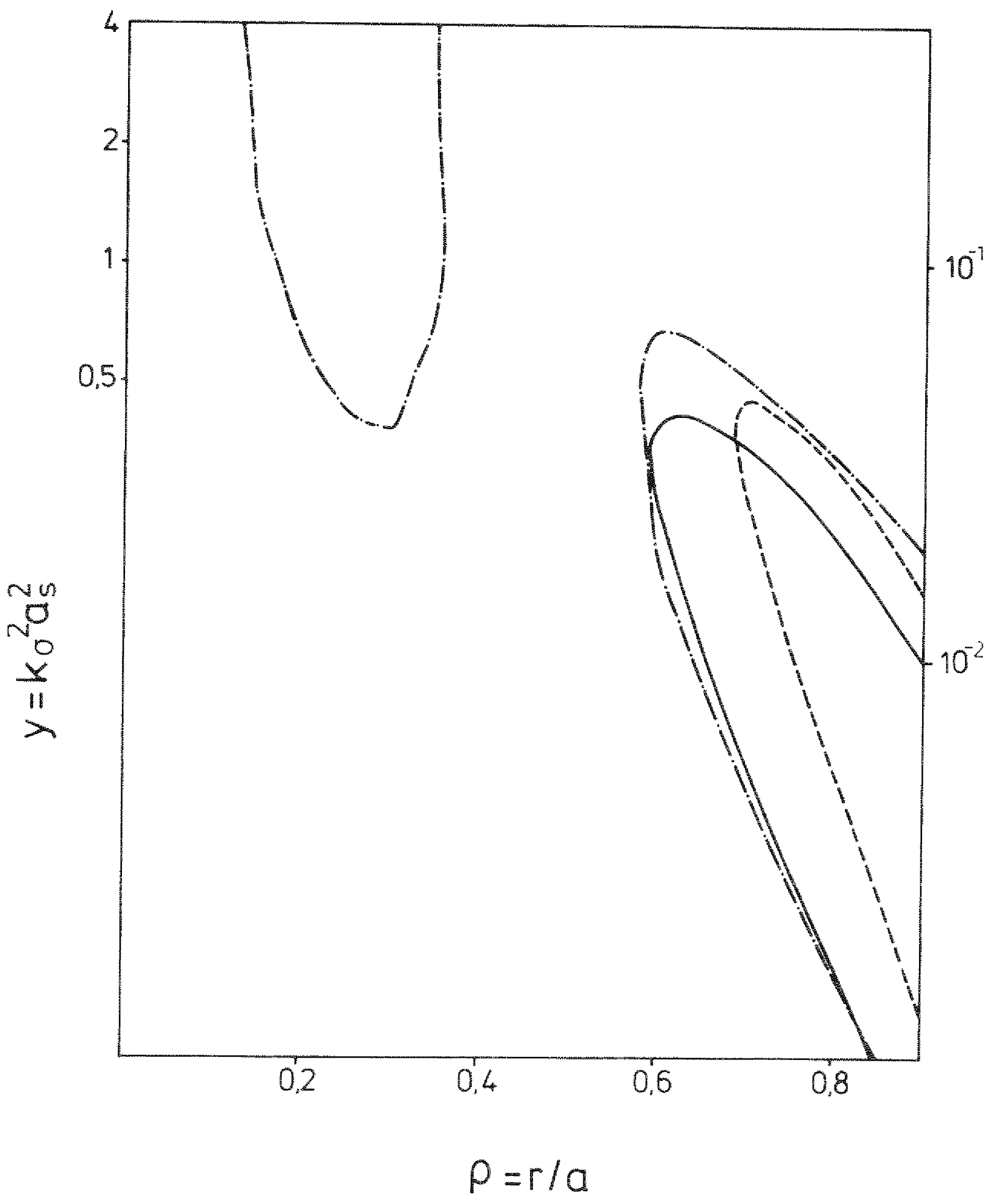




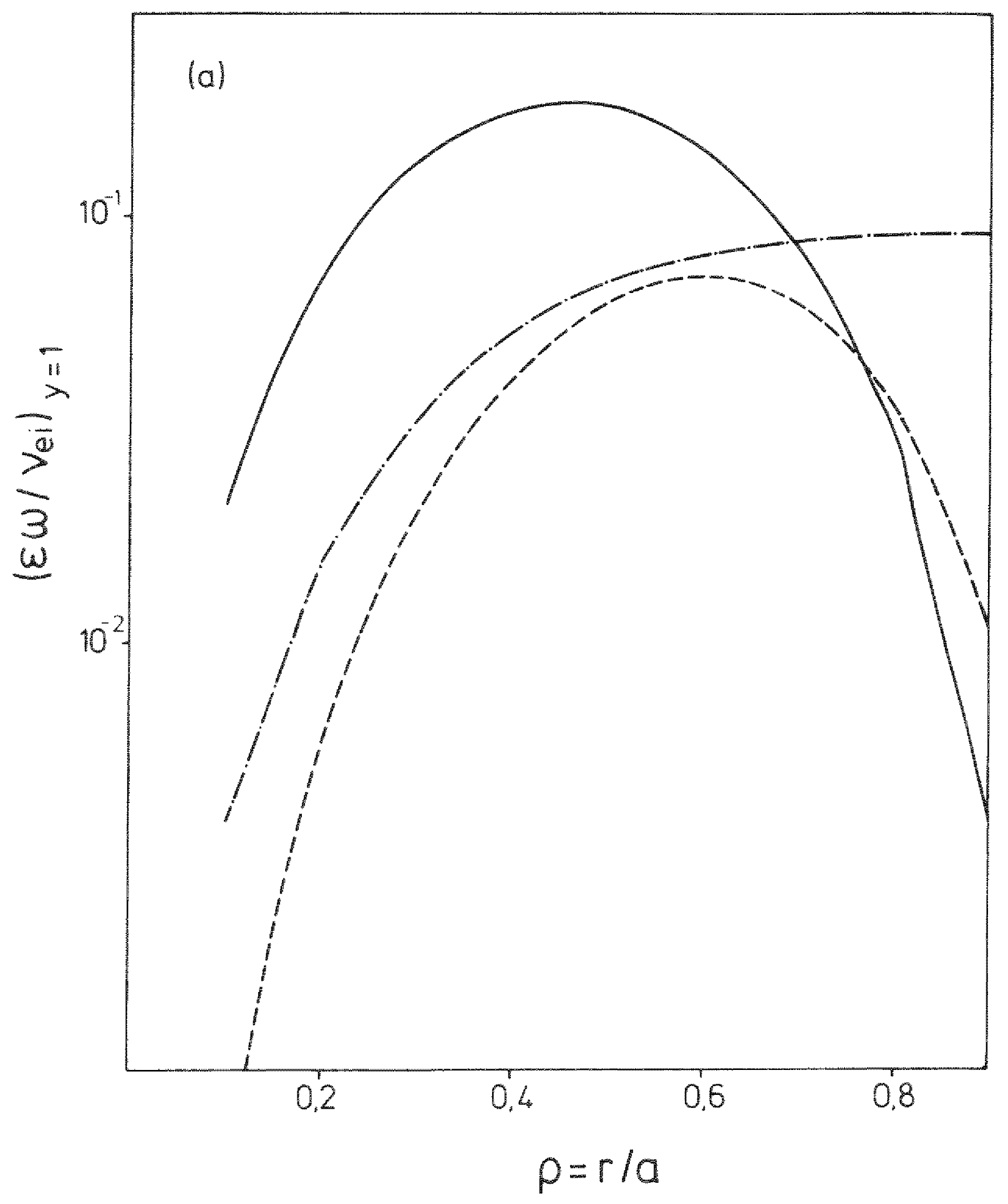




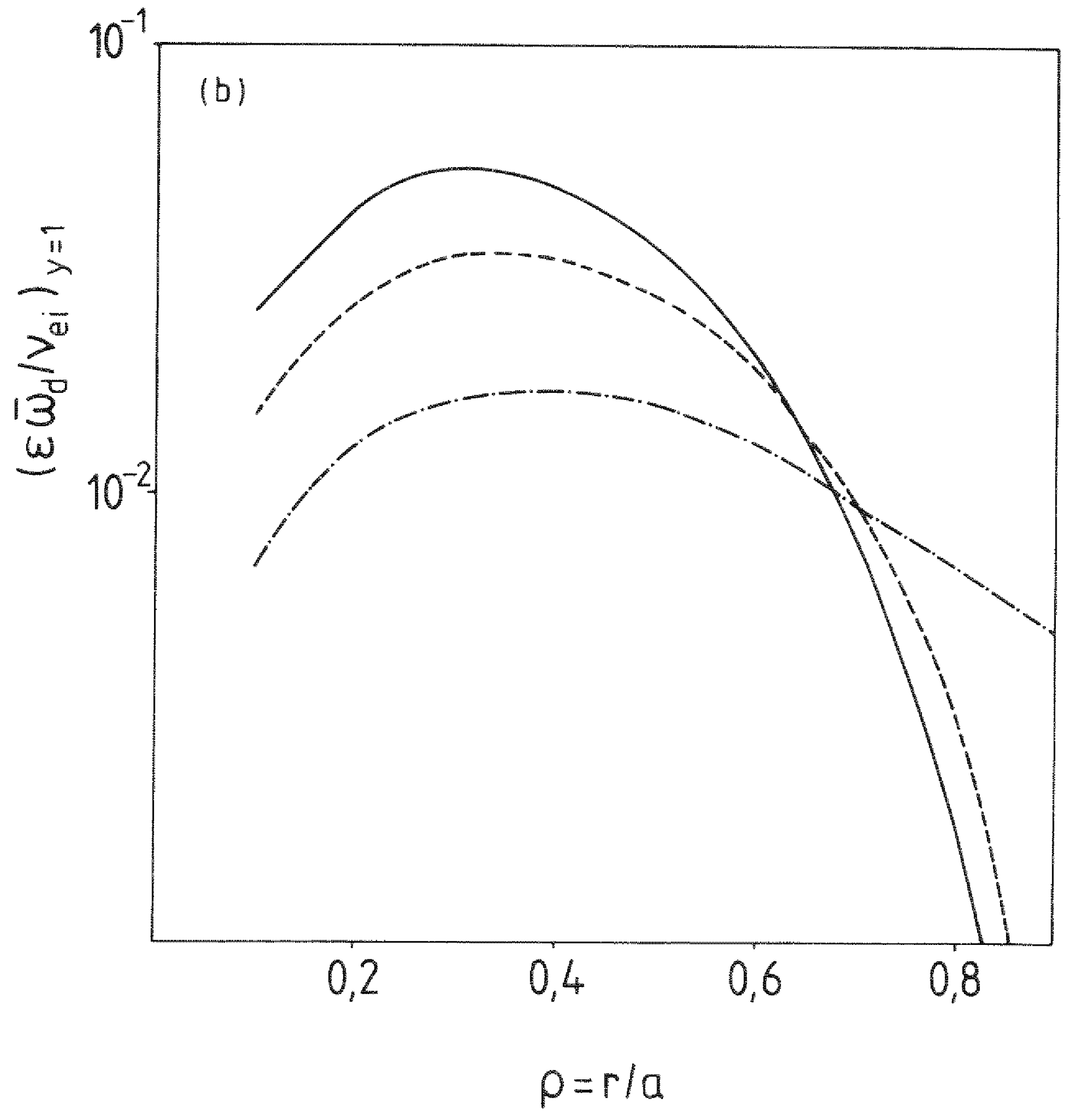




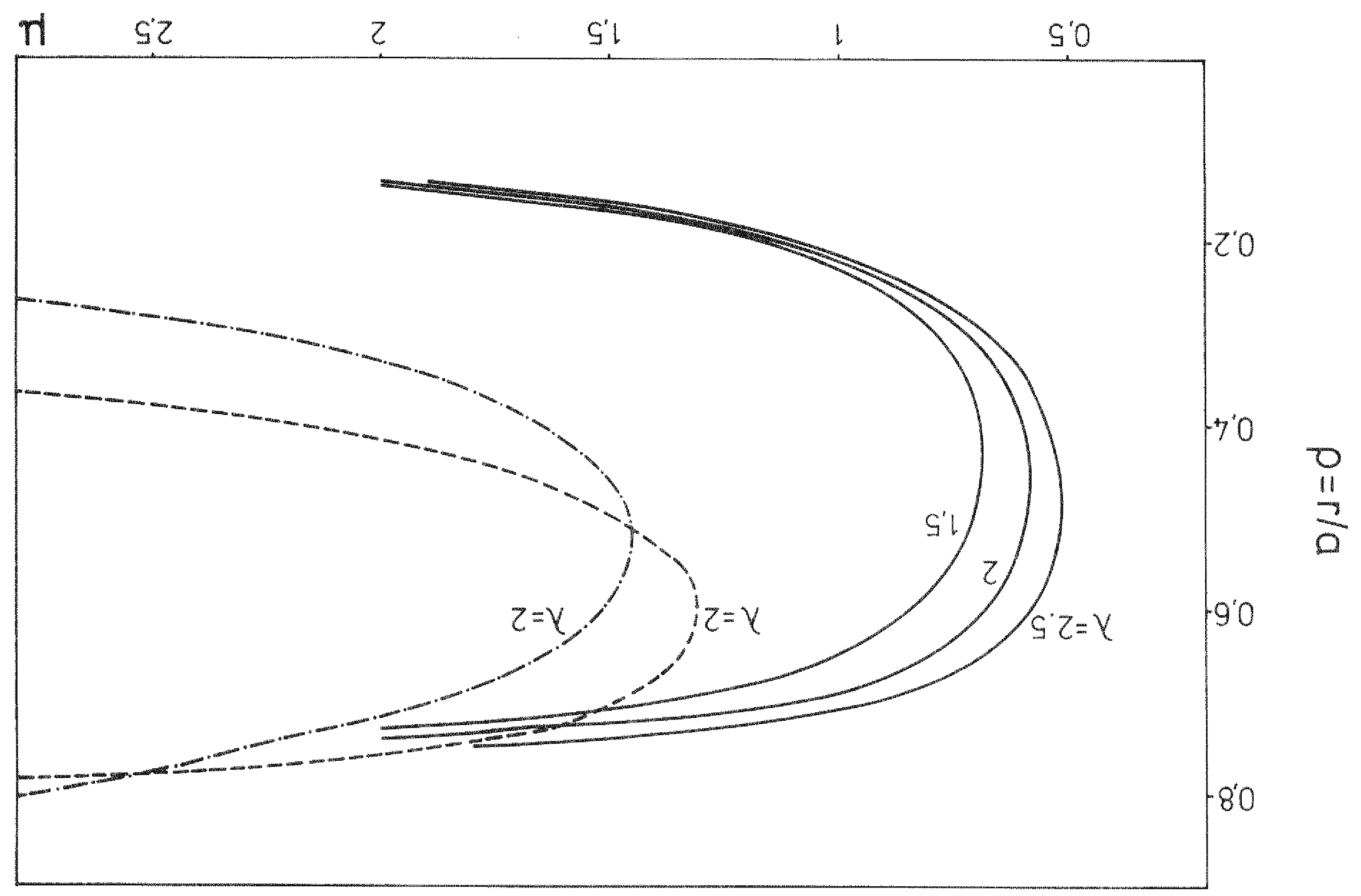




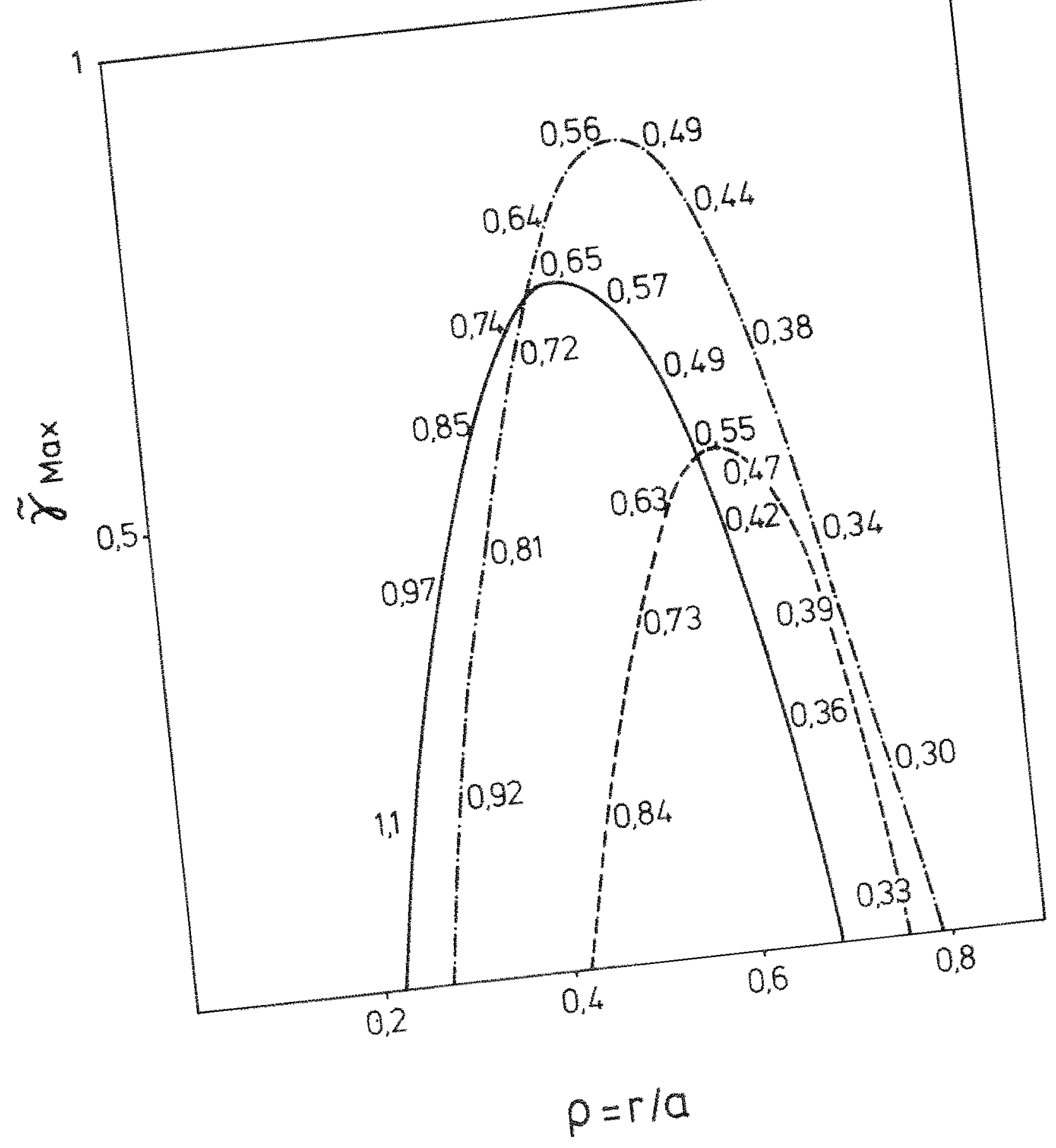




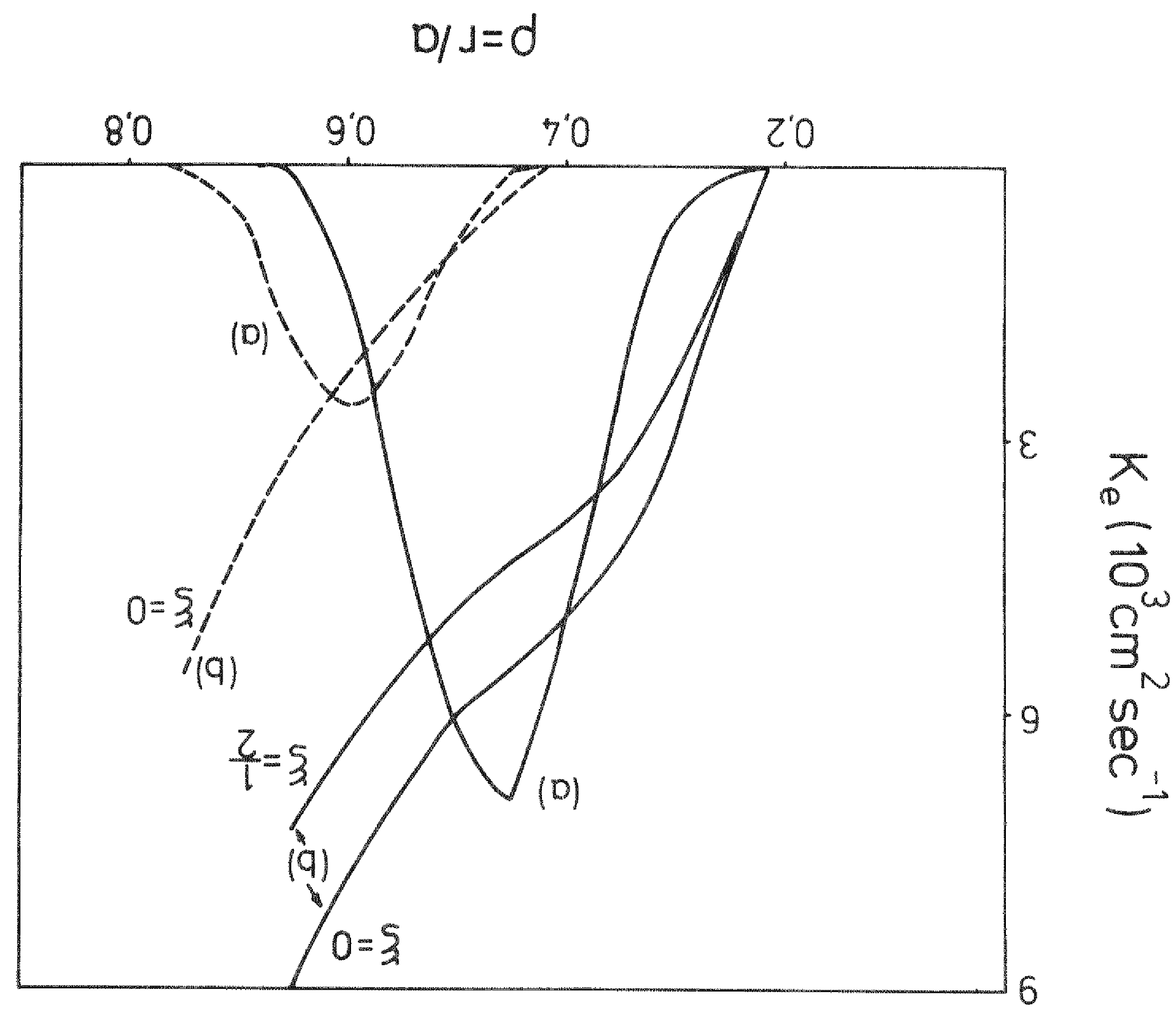




